\title{
Star clusters in the Magellanic Clouds
}

\section{Age-dating, classification, and spatio-temporal distribution of the SMC clusters $^{\star}, \star \star$}

\author{
P. K. Nayak ${ }^{1}$, A. Subramaniam ${ }^{1}$, S. Choudhury ${ }^{2}$, and Ram Sagar ${ }^{1}$ \\ ${ }^{1}$ Indian Institute of Astrophysics, Koramangala II Block, Bangalore 560034, India \\ e-mail: prasanta@iiap.res.in, purni@iiap.res.in \\ 2 Yonsei University Observatory, 120-749 Seoul, Republic of Korea
}

Received 2 November 2017 / Accepted 29 March 2018

\begin{abstract}
Aims. We aim to estimate the age and reddening parameters of already identified star clusters within the Small Magellanic Cloud (SMC) in a consistent way using available photometric data, classify them based on their mass and strength, and study their spatiotemporal distribution.

Methods. We have used a semi-automated quantitative method, developed in the first paper of this series (Paper I), to estimate the cluster parameters using the $V$ and $I$ band photometric data from the Optical Gravitational Lensing Experiment (OGLE) III survey. Results. We estimated parameters of 179 star clusters (17 are newly parameterised) and classified them into four groups. We present an online catalogue of parameters as well as cleaned and isochrone-fitted colour magnitude diagrams of 179 clusters.

We compiled age information of 468 clusters by combining previous studies with our catalogue, to study their spatio-temporal distribution. Most of the clusters located in the southern part of the SMC are in the age range $600 \mathrm{Myr}-1.25 \mathrm{Gyr}$, whereas, the clusters younger than $100 \mathrm{Myr}$ are mostly found in the northern SMC, with the central SMC showing continuous cluster formation. The peak of the cluster age distribution is identified at $130 \pm 35 \mathrm{Myr}$, very similar to the Large Magellanic Cloud (LMC) in Paper I.

Conclusions. We suggest that the burst of cluster formation at $130 \mathrm{Myr}$ is due to the most recent LMC-SMC interaction. $90 \%$ of the studied sample is found to have mass $<1700 M_{\odot}$, suggesting that the SMC is dominated by low mass clusters. There is tentative evidence for compact clusters in the LMC when compared to those in the Galaxy and the SMC. A progressive shifting of cluster location from the south to north of the SMC is identified in last $\sim 600 \mathrm{Myr}$. The details of spatio-temporal distribution of clusters presented in two videos as part of this study can be used as a tool to constrain details of the recent LMC-SMC interactions.
\end{abstract}

Key words. Magellanic Clouds - galaxies: star clusters: general - galaxies: star formation

\section{Introduction}

The Small Magellanic Cloud (SMC), located at a distance $(D)$ of $\sim 60 \mathrm{kpc}$ is a nearby dwarf galaxy to the Milky Way (MW) beyond the Large Magellanic Cloud (LMC, $D \sim 50 \mathrm{kpc}$ ). The SMC is classified as an irregular galaxy with a less pronounced bar and is known to be tidally disturbed because of its ongoing interaction with the LMC and the MW (Besla et al. 2010, 2012). The LMC and the SMC are enclosed within an extended body of diffuse HI gas that extends out to many tens of degrees across the sky, forming the Magellanic Stream and the leading arm (Wannier \& Wrixon 1972; Mathewson et al. 1974; Putman et al. 2003; Nidever et al. 2010). These features provide ample evidence of the MW-LMC-SMC interactions (Besla et al. 2010, 2012; Diaz \& Bekki 2011, 2012).

There have been several advances in understanding the interaction between these three galaxies over the last decade.

* Full Table 2 is only available at the CDS via anonymous ftp to cdsarc.u-strasbg.fr $(130.79 .128 .5)$ or via http://cdsarc.u-strasbg.fr/viz-bin/qcat?J/A+A/616/A187

$\star \star$ Two movies associated to Fig. 10 are available at https: //www . aanda.org
Diaz \& Bekki (2012) suggested that Magellanic Clouds (MCs) have undergone at least two pericentric passages about the MW during a $\sim 2$ Gyr bound association. On the other hand, the recent high precision measurement of proper motion of the MCs using the Hubble Space Telescope (HST) data suggests that either the MCs are undergoing their first passage near to the MW (Kallivayalil et al. 2013) or they are orbiting with a long period ( $>6 \mathrm{Gyr}$ ) around the Galaxy (Besla et al. 2010). The proper motion study of the LMC using HST data suggested that the MCs have just passed their pericentre (45 kpc from the Galactic centre) with apocentre to pericentre ratio of 2.5:1 with an orbital period of 1.5 Gyr (Kallivayalil et al. 2006). Diaz \& Bekki (2011, 2012) suggested that the SMC became a strongly interacting binary pair with the LMC only recently, suffering two strong tidal interactions $\sim 2 \mathrm{Gyr}$ ago and $\sim 250 \mathrm{Myr}$ ago. According to Besla et al. (2012), the SMC made close passages around the LMC at around $900 \mathrm{Myr}$ and $100 \mathrm{Myr}$ ago. These strong interactions between the MCs pulled out not only gas from the disc of the SMC, but also stars. The Gaia DR1 data revealed stellar tidal tails around both the Clouds and an almost continuous stellar bridge (Belokurov et al. 2017) connecting the two clouds. A significant number of Miras were found in the east of the LMC by Deason et al. (2017) using the first data released from Gaia 
mission and they inferred that these are likely to be stripped away from the SMC due to interaction with the LMC.

The close encounter between the MCs can also trigger star formation in both the clouds. Using Magellanic Clouds Photometric Survey (MCPS, Zaritsky et al. 2002, 2004) data, Harris \& Zaritsky (2004) showed that the burst of star formation happened at ages of 2.5, 0.4 and $0.06 \mathrm{Gyr}$ in the SMC. The burst timescales more or less coincide with the two past encounters between the MCs. The peaks at 2.5 and 0.4 Gyr also coincide with the star formation peaks in the LMC (Harris \& Zaritsky 2009). Any such triggered star formation can also lead to the formation of star clusters within a galaxy. Any propagation of cluster formation within a galaxy can also indicate interaction details. Hence, it will be useful to investigate the cluster formation history in the SMC along with their spatio-temporal distribution and correlate the peaks of cluster formation with the epoch of interaction.

A number of previous studies have been carried out to identify star clusters within the SMC. The recent and the most extensive study is presented by Bica et al. (2008, hereafter B08), where the authors listed $\sim 600$ star clusters in the SMC with their central coordinates, values of major and minor diameters, and position angle. However, the ages, reddening and mass of the clusters are not available in their catalogue. Using the MCPS data, Glatt et al. (2010, hereafter G10) estimated ages and reddening of 324 objects in the SMC, which include clusters and associations from the catalogue of B08. G10 found the age distribution of the clusters to have peaks at $160 \mathrm{Myr}$ and $630 \mathrm{Myr}$, and they suggested that the interaction between the MCs resulted in the formation of these peaks. Pietrzynski \& Udalski (1999, hereafter PU99) provided the age information of 93 well-populated SMC clusters using Optical Gravitational Lensing Experiment (OGLE) II survey data (Udalski et al. 1998). Chiosi et al. (2006) presented the ages of 311 clusters younger than 1 Gyr. The authors used two sets of data for their analysis: OGLE II for the SMC disc and the data obtained from the ESO $2.2 \mathrm{~m}$ telescope for the region around NGC 269, located in the south-east end of the disc. These authors found that the age distribution of clusters showed an enhancement between 15 and 90 Myr.

Studies by Piatti et al. (2005, 2007a, b, c, 2008, 2011), Piatti (2011), and Maia et al. (2012) made use of deep Washington photometric data concerned primarily intermediate and old clusters in the SMC. Mighell et al. (1998), Glatt et al. (2008), Girardi et al. (2013) studied intermediate and old clusters using photometric data obtained from the HST. Age, metal abundance, and positional data of 12 star clusters were presented by Crowl et al. (2001). Ages of 15 intermediate to old star clusters were determined Parisi et al. 2014. Dias et al. (2014, 2016) derived age, metallicity, reddening and distance for star clusters in the SMC west halo. Recently Piatti et al. (2015) studied 51 star clusters in the eastern outskirt of the SMC and in the bridge region using the VMC survey. Based on the CMDs they defined 15 catalogued clusters to be possible non-genuine aggregates.

Despite several past studies, the number of SMC clusters with age information is only about $50 \%$ of that listed by B 08 . Also, as mentioned by the authors, the list of detected clusters in the SMC is still incomplete (mostly restricted by poor detection limit). Therefore, to understand cluster formation history in detail and the effect of LMC-SMC-MW interactions on cluster formation, one has to determine the ages of already catalogued clusters, at the same time new clusters have to be identified using relatively deeper and large scale photometric data.

In this study, we have tried to estimate the age and reddening of the already identified star clusters by B08. The usual method of estimating the age of a cluster is by visual fitting of isochrones over the cluster CMD, which can produce a systematic error in the age estimation from cluster to cluster. At the same time, when cluster sample is large (more than hundred), it is a tedious job to fit isochrones to each cluster. Therefore, we have used the semiautomated quantitative method developed by Nayak et al. (2016, hereafter Paper I) to estimate the age and reddening. Using this method we also quantify the error in age.

The SMC has long been known to host primarily rich clusters. Nevertheless, recent studies suggested that it also contains poor clusters (Piatti \& Bica 2012) and hosts clusters with a wide range of masses (Hunter et al. 2003). Using integrated colour in UBVR passbands and evolutionary models, Hunter et al. (2003) estimated masses of 191 clusters in the SMC having a range between $10^{2}$ to $10^{6} M_{\odot}$. Kontizas et al. (1982) calculated masses of 20 clusters in the SMC using King (1962) model and found that derived masses are about ten times smaller than those in our Galaxy. Using archival HST snapshot data, Mackey \& Gilmore (2003) determined masses of ten rich clusters from their surface brightness profiles. The estimated mass of those clusters range from $10^{3.6}$ and $10^{5.5} M_{\odot}$. Recent study by Maia et al. (2014) has provided masses of 29 young and intermediate clusters within a range of 300-3000 $M_{\odot}$.

So far, there has not been any study to systematically classify the clusters based on their mass. Searle et al. (1980) classified 61 star clusters in the MCs as type I to VII based on four colour photometry of integrated light. On the other hand, there are well known classification schemes for Galactic Open Clusters (Trumpler 1930) based on the degree of central concentration of stars, the range in luminosity of the members, the number of stars contained in the cluster and necessary conspicuous properties (Ruprecht 1966). In this paper, we have tried to estimate the mass range of the SMC clusters and classify them based on their mass as well as richness. Estimation of the mass and age of cluster sample will help in understanding cluster formation, evaporation, and dynamical evolution of the cluster system.

Thus, the aims of this study are: (1) to estimate age and reddening of already identified star clusters of the SMC in a consistent way using available photometric data and increase the sample of well-studied clusters (2) to classify the SMC clusters based on their mass/richness (3) to study the spatio-temporal distribution of the SMC clusters.

The rest of the paper is arranged as follows: In Sect. 2 we have mentioned about the data used for this study, followed by analysis in Sect. 3. Sect. 4 presents error estimation. Estimation of cluster mass and the classification scheme are described in Sect. 5. Results of this study are presented in Sect. 6, followed by the summary in Sect. 7 .

\section{Data}

We have used $V$ and $I$ photometric data from the OGLE III (Udalski et al. 2008) survey to identify star clusters, which are listed in B08. The OGLE III observations were carried out at the Las Campanas Observatory, Chile, between June 2001 and January 2008, with the 1.3-m Warsaw telescope equipped with second generation mosaic camera (Udalski 2003) consisting of eight SITe $2048 \times 4096$ CCD detectors with pixel size of $15 \mu \mathrm{m}$, which corresponds to $0 .^{\prime \prime} 26$. The field of view of the telescope is approximately $35 \times 35 \operatorname{arcmin}^{2}$ on the sky. The survey covered a total area of about 14 square degrees in the sky around the SMC centre and produced a catalogue of $V$ and $I$ magnitudes of about 6.2 million stars (Udalski et al. 2008). The completeness of the 
photometry is better than $75 \%$ in I band and $85 \%$ in $V$ band for 20 mag for crowded regions.

In this study, we have estimated the parameters (age, reddening, mass and richness) of star clusters that can be affected by photometric incompleteness of the data. Photometric incompleteness will lead us to a wrong determination of cluster richness. A rich cluster located in the crowded field can appear as poor cluster due to photometric incompleteness. The luminosity function (LF) of a cluster will also be biased due photometric incompleteness and it will guide to a wrong estimation of mass function as well as mass of the clusters. The method used here to estimate age and reddening of clusters will also be affected by photometric incompleteness as well as photometric error. Therefore, we have considered stars as having photometric errors $\leq 0.15 \mathrm{mag}$ in $V$ and $I$ bands with photometric completeness more than $90 \%$ even in the most crowded region to construct LF. We used the full data when it comes to plotting the colour magnitude diagrams (CMDs).

\section{Analysis}

\subsection{Cluster sample}

We adopted the most extensive SMC cluster catalogue by B08 as a reference and identified 492 star clusters located well within the OGLE III observed region, mentioned in the last section. We define the radius of clusters to be $1 / 4$ (major + minor) diameter, the values of which are given in B08 catalogue. The estimated cluster radii are found to range from 0.07 to 1.70 on the sky, with physical sizes corresponding to a range of $\sim 1.22-29.6 \mathrm{pc}$. We extracted data of the cluster regions (stars within the cluster radius) from the OGLE III catalogue along with a few arcmin field around them. These data are used for further analysis. Further details of the extraction are described in Paper I.

A star cluster is defined as a gravitationally bound system of a group of stars and can be observed as a density enhanced region with respect to its surrounding field region. The observed cluster region not only consists of cluster members but also has foreground and background field stars. The SMC hosts a good number of rich as well as poor clusters, located in a varied range of stellar density environments. The fundamental features of a cluster which can be used to estimate the reddening, age and distance are the main-sequence (MS) and the location of MS turn-off in the CMD. So, field star removal is necessary to define the cluster sequence and for a better estimation of cluster parameters. We first identified the clusters that are located in an environment with varying field star density. The estimated number of stars within the cluster radius is denoted as $n_{c}$. In order to estimate variation in the field star density, we chose four annular regions (each of equal area as the cluster region), of inner radii $0.5,1 . .0,1.5$, and 2.'0 larger than the cluster radius and counted the number of stars in each annular region. The number of field stars, which is an average of estimation from the four field regions $\left(n_{f}\right)$ is considered to be contaminating the cluster region. Standard deviation $\left(\sigma_{f}\right)$ about this average indicates the variation in the field star density. The number of cluster members $\left(n_{m}\right)$ or strength of the cluster is defined as $n_{m}=n_{c}-n_{f}$. Many clusters are found to have $\sigma_{f} \sim n_{m}$, suggesting that the cluster strength is similar to the fluctuation in the field star distribution. Then, we proceeded to exclude clusters which have the following properties:

(i) We estimated the fractional standard deviation as $\sigma_{f} / n_{f}$, to quantify the variation in field star counts. We excluded those clusters where variation in the field stars count is greater than or equal to $50 \%$ of the average count, that is, $\sigma_{f} / n_{f} \geq 0.5$. With this criteria, we excluded 48 star clusters.

(ii) The variation in the field star counts will propagate as an error when we estimate the strength of the cluster. The error associated with the estimation of $n_{m}$ can be defined as

$e=\left|\left(n_{c}-\left(n_{f}-\sigma_{f}\right)\right)-\left(n_{c}-\left(n_{f}+\sigma_{f}\right)\right)\right|$,

which is basically the difference between the maximum and the minimum values of $n_{m}$, for a $\sigma_{f}$ deviation in the field star distribution. For crowded field regions, there is a possibility that $\sigma_{f}$ is high and so will be the value of $e$. In order to remove clusters where the error itself is greater than the number of stars in the cluster, we calculated the fractional error as $e / n_{m}$ and excluded clusters with $e / n_{m} \geq 1$. Based on this criteria, we excluded 126 clusters from our sample.

The number of clusters remaining in the sample, after implementing the above two cut-off criteria is 337. Of these clusters, five are relatively rich clusters $\left(n_{m}>400\right)$ and we have excluded them from our analysis as they are already well studied using better observational data. Thus, we proceeded with a sample of 332 clusters to decontaminate their cluster CMDs. This exercise also points out that $\sim 30 \%$ of the clusters are located in regions with significant variation in field star density. In Paper I, we found that about $20 \%$ of clusters are located in similar environments within the LMC.

We constructed $(V, V-I)$ CMDs for cluster and field regions to compare and decontaminate the cluster region from field stars using a statistical process. For the details of this process, we direct the readers to Sect. 3.2 of Paper I.

\subsection{Semi-automated quantitative method}

We have adopted the semi-automated quantitative method developed in Paper I, to estimate cluster parameters accurately and consistently. We also quantified error using this method. The method is applied to all the 332 clusters to estimate reddening and age.

The primary steps involved in the method are to:

(a) Identify the MS in the cleaned cluster CMD and construct the MS luminosity function (MSLF).

(b) Identify the MS Turn-off (MSTO) from the MSLF and estimate the corresponding apparent magnitude and colour.

(c) Estimate the reddening from the $(V-I)$ colour of the MSTO.

(d) Estimate the absolute magnitude of the MSTO after correcting for reddening and distance.

(e) Estimate the age using age-magnitude relation derived using Marigo et al. (2008, hereafter M08) isochrones.

The above steps are described in detail below.

(a) We consider stars brighter than $21 \mathrm{mag}$ in $\mathrm{V}$ and bluer than 0.5 mag in $(V-I)$ colour as the MS stars. To construct the MSLF, the magnitude axis is binned with a bin size of 0.2 mag. The brightest bin with a minimum number of stars $(\eta)$ is identified as the bin corresponding to the MSTO. The mean $V$ magnitude of the brightest bin is considered as turnoff $V\left(V_{\mathrm{TO}}\right)$ magnitude. The MSTO bin (which is likely to be the brightest bin of the MSLF) needs to be identified from the MSLF using statistically significant value of $\eta$ so that it excludes blue super giants. The value of $\eta$ will depend mainly on richness as well as age of the cluster. Two clusters which are similar in richness, but with different age will have different MSTO bin with different $\eta$. The MSTO bin will be less populated for a younger cluster than the older one with similar richness. Two clusters with same age but a different 
Table 1. Grouping and classification of clusters based on their richness $\left(n_{m}\right)$ and mass range $\left(M_{c}\right)$.

\begin{tabular}{lcccccc}
\hline \hline Group no. & Range of $n_{m}$ & $\eta$ & $\eta_{\text {simulated }}$ & $N_{\text {total }}$ & Mass range $\left(M_{\odot}\right)$ & Classification \\
\hline I & $6<n_{m} \leq 30$ & 2 & 1.23 & 94 & $<800$ & very poor \\
II & $30<n_{m} \leq 100$ & 3 & 2.88 & 69 & $800-1700$ & poor \\
III & $100<n_{m} \leq 200$ & 5 & 6.16 & 13 & $1700-3500$ & moderate \\
IV & $200<n_{m} \leq 300$ & 10 & 10.27 & 2 & $3500-5000$ & moderate \\
V & $300<n_{m} \leq 400$ & 14 & 14.38 & 1 & $>5000$ & rich \\
\hline
\end{tabular}

number of cluster stars will also have different values of $\eta$ for their MSTO bin. So, the identified bin and the number of stars in the bin are dependent on the richness/age of the cluster. Here, the only known parameter is the richness (total number of cluster stars) of the cluster, as we are yet to estimate their age. Therefore, we have grouped the clusters according to their strength, similar to Paper I, and considered similar $\eta$ value corresponding to each group to identify the MSTO bin. In the next section, we have briefly described the calibration procedure of $\eta$. We refer to Sect. 3.4 of Paper I for more details. After classifying the group number, strength of clusters and the $\eta$ value corresponding to each group are tabulated in Table 1.

(b) Once we have identified the $V_{\mathrm{TO}}$, the next task is to estimate the colour of the MSTO. The colour of the MSTO can be identified as the peak in colour distribution near the MSTO. To estimate the peak colour of the MSTO, a strip parallel to the colour axis with a width of $0.6 \mathrm{mag}$ about $V_{\text {TO }}$ is considered ( $V_{\mathrm{TO}}+0.4 \mathrm{mag}$ to $V_{\mathrm{TO}}-0.2 \mathrm{mag}$ ). This is to ensure that we have a statistically significant number of stars near the MSTO. For the clusters with $n_{m} \leq 100$, a width of $0.8 \mathrm{mag}$ is considered (given by $V_{\mathrm{TO}}+0.6 \mathrm{mag}$ to $V_{\mathrm{TO}}-0.2 \mathrm{mag}$ ). The choice for width of the strip does not affect the position of the peak colour, as the isochrones for younger ages are almost vertical to the colour axis near the MSTO. This strip is binned in colour with a bin size of 0.1 mag to estimate the distribution of stars along the colour axis. The distribution is found to have a unique peak (in most of the cases) with asymmetric wings. The mean colour of the bin corresponding to this unique peak is chosen as the apparent colour, $(V-I)_{\mathrm{app}}$, of the MSTO.

(c) The reddening of the cluster is defined as the difference between the apparent and absolute colour of the MSTO. To begin with, we have adopted $A_{V}=0.46 \mathrm{mag}$ (Zaritsky et al. 2002) and distance modulus of $\mathrm{DM}=18.90 \mathrm{mag}$ for the SMC (Storm et al. 2004). If $\mathrm{M}_{V}$ is the absolute magnitude of the MSTO, then assuming a distance modulus and an average value of extinction $\left(\mathrm{A}_{V}\right)$ for the cluster, the apparent magnitude $\left(V_{\mathrm{TO}}\right)$ is related to $\mathrm{M}_{V}$ as

$M_{V}=V_{\mathrm{TO}}-\mathrm{DM}-A_{V}$.

The estimated value of $\mathrm{M}_{V}$ is cross-matched with the absolute $\mathrm{V}$ magnitude of MSTOs from the isochrones table of M08 for a metallicity of $Z=0.004$ for the SMC. The $(V-I)$ colour corresponding to the closest match of absolute $\mathrm{V}$ magnitude of MSTO gives the true colour for the MSTO, $(V-I)_{0}$. The reddening $(E(V-I))$ for the cluster is then given as

$E(V-I)=(V-I)_{\mathrm{app}}-(V-I)_{0}$.

(d) The extinction for the cluster region is estimated as, $\mathrm{A}_{V}=$ $2.48 \times E(V-I) \quad($ Nikolaev et al. 2004). The extinction corrected value of $\mathrm{M}_{V}$ of the MSTO is then calculated again

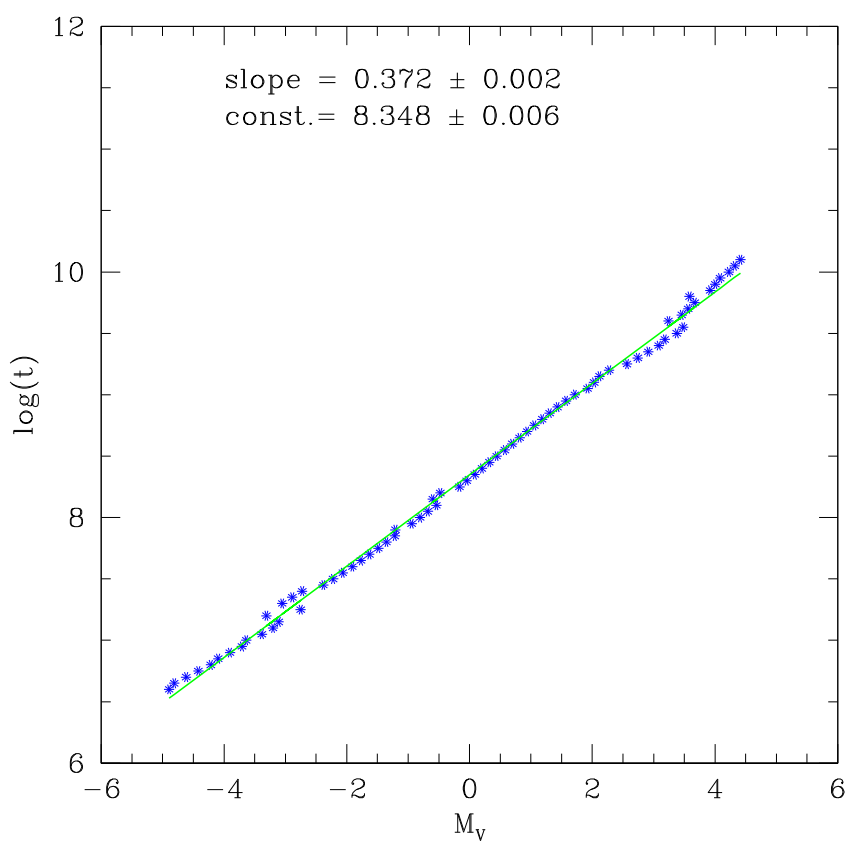

Fig. 1. Relation between the absolute turn-off $V$ magnitude $\left(M_{V}\right)$ and age, within the range $\log (t)=6.2-10.2$ for M08 isochrones. A straight line (green) fitted through the points is also shown.

by using this value of $\mathrm{A}_{V}$ in Eq. (2). The values are found to be invariant even after a couple of iterations. The method used here is similar to that adopted by Indu \& Subramaniam (2011), for estimating the reddening of field regions.

(e) Figure 1 shows relation between the absolute magnitude $M_{V}$ of the MSTO and their corresponding ages $(\log (t))$ for M08 isochrones. The relation is found to be linear and is given as:

$$
\log (t)=0.372( \pm 0.002) M_{V}+8.348( \pm 0.006) .
$$

The extinction corrected $\mathrm{M}_{V}$ derived in step (d), is used in the above relation to estimate the ages of the clusters.

Once we had estimated the parameters, we over plotted isochrones on each cluster CMD of corresponding age, after correcting for estimated reddening and extinction. We visually checked all the CMDs for any improper estimation of parameters. If required, we adjusted the parameters to improve the isochrone fit. As we have finalised the parameters after visual inspection, the method is termed as semi-automated quantitative method.

This method primarily depends on the unique identification of the MSTO. As mentioned earlier, it is a function of age and richness of the cluster. Due to the photometric limit of OGLE III data (21 magnitude in $\mathrm{V}$ band), we have restricted the method to clusters with MSTO magnitude $\left(V_{\mathrm{TO}}\right)$ brighter than 19 mag for reliable estimation of clusters parameters. There are 189 clusters with $V_{\text {TO }} \leq 19$ mag. We visually checked the cluster CMDs 
with over plotted isochrones. We found that the isochrones fitted very well for 62 clusters $(32.8 \%)$ and a small correction in age and/or reddening were required for 75 clusters $(39.7 \%)$. The remaining 52 clusters $(27.5 \%)$ have ambiguous cluster sequence. To identify the cluster sequence we decontaminated the cluster region for these 52 clusters with two more field regions and over-plotted all decontaminated CMDs. The sources which have not been removed for at least two different decontamination processes were considered as a cluster member. Out of the 52 clusters, 12 were found to show prominent cluster features, and required minor modification in age or/and reddening, to the automated estimation. Whereas, 40 clusters were found to have no clear feature in the CMD prohibiting any reliable estimation of parameters. Among these 40 clusters, four are found to be in common with the cluster candidates identified by Piatti et al. (2015).

For star clusters in the LMC (Paper I), the semi-automated quantitative method worked well for more than $80 \%$ of the total sample. The reason for reduced success rate in the case of the SMC is probably due to less number of cluster members $\left(n_{m} \leq 30\right)$, resulting in sparsely populated CMDs and MS.

To increase the number of parameterised clusters, we also inspected the 143 cases (out of 337) with $V_{\text {TO }}>19$ mag. After the visual inspection of their CMDs, 30 clusters are found to have reliable estimation (including a minor correction in estimated age and/or reddening with respect to automated estimation for a few). Thus, we were able to estimate the parameters of a total 179 clusters within the SMC.

\subsection{Calibration procedure of $\eta$}

The value of $\eta$ depends on richness and age of the cluster. Limited by the photometric depth, OGLE III data is ideal to estimate the ages of mainly younger clusters (upto few hundred Myr) and $\eta$ will not depend much on this small age spread, as we have seen in Paper I. Therefore, it is necessary to fix the value of $\eta$ for different groups of clusters to estimate the parameters. After we grouped the clusters based upon their strength, we looked for the clusters from each group whose parameters are already estimated by G10. We then estimated age and reddening of those clusters from a particular group for a range of $\eta$ values. We compared our age and reddening estimations with that of G10 for all $\eta$ values. We chose the $\eta$ value for which we found that deviations in the estimated parameters (age and reddening) are least and there are no systematic deviations with respect to G10's estimations. This is the way we calibrated the $\eta$ value for each group of clusters.

We also calibrated the $\eta$ value by generating synthetic CMDs and comparing it with observed CMDs. In this study, we found that ages of the SMC clusters peak at $\sim 100 \mathrm{Myr}$ and reddening peaks between 0.10 and 0.20 . We took age to be $100 \mathrm{Myr}$ and reddening value as 0.15 to produce synthetic CMDs. In this analysis, we used Padova isochrone model (Marigo et al. 2008) and Salpeter's mass function (Salpeter 1955). We produced synthetic CMDs by populating stars in the main sequence (MS) for observed ranges of $V$ and $I$ mag. We have also taken care of photometric incompleteness while generating synthetic CMDs. We calculated the number of stars present in the turn-off bin, which is nothing but the value of $\eta$, for different groups $(I-V)$ of clusters. We have run the simulation for multiple iteration with different initial normalising star-counts. We have also run the simulation for another two age values (200 and $300 \mathrm{Myr}$ ). We found that the $\eta$ values estimated from the above two methods match very closely and are tabulated in Table 1.

\section{Error estimation}

We have calculated the errors associated with the estimated age and reddening using the method of propagation of errors, adopted from Paper I. The error in estimating the reddening depends upon the photometric error and binning resolution along the colour axis, and this error will propagate to age estimation. The error associated with the age estimation depends on the errors in estimating extinction and absolute magnitude, and binning resolution along the magnitude axis. We have also considered the effect of distance spread in the SMC on age estimation. The SMC has a large range of line of sight depth of the SMC from $670 \mathrm{pc}$ to $9.53 \mathrm{kpc}(0.025$ to $0.34 \mathrm{mag}$; Subramanian \& Subramaniam 2009). In our error analysis we considered the maximum depth of the SMC $(9.53 \mathrm{kpc})$. As the reddening is estimated using the stars in the upper MS and the photometric error is very small $(\leq 0.05)$ in the upper MS, the effect of photometric error in estimating reddening could be neglected. Thus, the error in the estimated reddening, $E(V-I)$ is chosen to be same as the bin size, 0.1 magnitude. Errors in the estimation of extinction and age are given by the following relations

$$
\begin{aligned}
& \sigma A_{V}=2.48 \sqrt{\sigma(V-I)^{2}+(V-I)_{\mathrm{bin}}^{2}} \\
& \sigma M_{V}=\sqrt{\sigma V^{2}+V_{\mathrm{bin}}^{2}+\sigma A_{V}^{2}+\sigma(D M)^{2}} \\
& \sigma(\mathrm{age})=\text { constant } \times \sigma M_{V}
\end{aligned}
$$

where $V_{\text {bin }}$ and $(V-I)_{\text {bin }}$ are half the bin sizes used for magnitude and colour binning, $\sigma M_{V}$ is the error in absolute magnitude, $\sigma A_{V}$ is the error in the estimated extinction, $\sigma(D M)^{2}$ is the uncertainty in the distance modulus due to maximum line of sight depth in the SMC and $\sigma($ age $)$ is the error in estimated age in $(\log (t))$. The maximum error in estimated age is 0.25 .

The studies of intermediate and old SMC clusters by Parisi et al. (2009) suggested that metallicity of the SMC clusters ranges from -0.60 to -1.30 dex with a mean of -0.96 dex. Therefore, we have also examined the effect of metallicity on estimated age. We derived $\mathrm{M}_{V}$ vs $\log ($ age $)$ relation for three different metallicities $(Z=0.001,0.004,0.008)$ and found that the variation in the slope and the y-intercept are in second and third decimal place respectively. The error in the age estimation varies from 0.24 to 0.26 in log scale due above mentioned metallicity range.

\section{Estimation of mass range}

We have divided the total cluster sample into five different groups (group I-group V) based on their strength $\left(n_{m}\right)$ using the same criteria as in Paper I. In Table 1, the group numbers and corresponding range of cluster strength are listed in Columns 1 and 2 respectively. Column 3 gives the list of $\eta$ values for different groups, which helps to identify turn-off magnitude in the cluster CMD. Cluster strength not only gives information about richness of the cluster but also indicates the mass of the cluster. We have tried to estimate the mass range for clusters corresponding to each group. Then, we classified the clusters based on their mass range using similar classification scheme as in Paper I.

In our sample, we find that most of the clusters are younger than $300 \mathrm{Myr}$ and the age distribution peaks at around $100 \mathrm{Myr}$. Hence, we assumed a typical age of $100 \mathrm{Myr}$ and used the value of $\eta$ to estimate the mass $\left(M_{c}\right)$ range of clusters. We constructed synthetic CMDs using M08 isochrones, for a mass range of 0.1-15.0 $M_{\odot}$. We assumed the mass function to be Salpeter's 
Table 2. Sample of the complete catalogue is presented.

\begin{tabular}{lccccccccc}
\hline \hline Star cluster & $\begin{array}{c}\text { RA } \\
(\mathrm{h} \mathrm{m} \mathrm{s})\end{array}$ & $\begin{array}{c}\text { Dec } \\
\left({ }^{\circ}{ }^{\prime \prime}\right)\end{array}$ & $\begin{array}{c}\text { Radius } \\
\left({ }^{\prime}\right)\end{array}$ & $E(V-I)$ & $\log (t)$ & $\log \left(t_{\mathrm{G} 10}\right)$ & $\log \left(t_{\mathrm{PU} 99}\right)$ & $\log \left(t_{\mathrm{C} 06}\right)$ & Group \\
\hline B6* & 02757 & -742402 & 0.30 & 0.10 & 8.25 & - & - & - & I \\
K9 & 03000 & -732245 & 0.60 & 0.17 & 8.60 & 8.70 & - & - & II \\
HW8 & 03346 & -733759 & 0.85 & 0.11 & 7.90 & 8.00 & - & - & II \\
NGC176 & 03558 & -730958 & 0.60 & 0.12 & 7.64 & 8.20 & - & - & II \\
HW11 & 03733 & -733643 & 0.65 & 0.15 & 8.20 & 8.50 & 8.4 & 8.4 & II \\
L19 & 03742 & -735430 & 0.85 & 0.16 & 8.70 & - & $>9.0$ & 8.9 & IV \\
B14 & 03837 & -734821 & 0.26 & 0.10 & 8.25 & 8.65 & - & 7.9 & I \\
HW12 & 03851 & -732227 & 0.40 & 0.09 & 8.45 & 8.70 & - & 8.7 & I \\
H86-48 & 03856 & -732432 & 0.22 & 0.07 & 8.30 & - & - & 8.0 & I \\
SOGLE6 & 03933 & -731037 & 0.40 & 0.17 & 8.70 & 8.65 & - & - & I \\
\hline
\end{tabular}

Notes. The table contains the name of the cluster, central coordinates (RA and Dec) as given in B08, size of the cluster taken from B08, estimated reddening and age, in Cols. 1-6 respectively. Columns 7-9 contain the earlier estimations of ages by $\mathrm{G} 10\left(\log \left(t_{\mathrm{G} 10}\right)\right), \mathrm{PU} 99\left(\log \left(t_{\mathrm{PU} 99}\right)\right)$ and $\left(\log \left(t_{\mathrm{C} 06}\right)\right)$. The last column contains the designated group number $(I-V)$.

mass function (Salpeter 1955) and included observational errors. To reduce statistical fluctuation because of low $\eta$ value, we simulated the synthetic CMD for a large number of stars $\left(\sim 10^{6}\right)$ and scaled for the value of $\eta$ in the MSTO bin. The total mass of the cluster is estimated using the scaling factor. We found that the clusters in group I have $M_{c}<800 M_{\odot}$ and we classify them as very poor. Clusters in group II have $M_{c}$ in the range $\sim 800-$ $1700 M_{\odot}$ and classified as poor. Groups III and IV have been classified as moderately rich clusters with $M_{c}$ range $\sim 1700$ $5000 M_{\odot}$ and the clusters in the group $\mathrm{V}$ as rich clusters with $\mathrm{M}_{c}>5000 M_{\odot}$. In Col. 5 of Table 1, mass range of clusters for different groups are listed and in Column 6 the classification of clusters are noted. The total number of clusters $\left(N_{\text {total }}\right)$ in each group is listed in Col. 4.

The estimated mass ranges for different groups indicate that the SMC consists of clusters with a large mass range, similar to the LMC star clusters (Paper I). The classification of clusters based on mass range will help us to understand various properties, like formation and evolution of clusters, which depend on its mass. It will also help us in understanding the dissolution of star clusters in various groups in the SMC. Our estimated mass ranges match well with that of Maia et al. (2014) and Hunter et al. (2003). We have also compared the mass range of the SMC clusters with that of the open clusters in the Galaxy. We find that the clusters near solar neighbourhood have mass range (Lamers et al. 2005) similar to that in the SMC. Piskunov et al. (2008) studied 650 Galactic open clusters with mass range $50 M_{\odot}$ to $10^{5} M_{\odot}$. Thus, SMC consists of clusters with a large mass range which is similar to Galactic open clusters. In our sample, about $50 \%$ of the clusters belong to the very poor group, suggesting the presence of a large fraction of very low mass clusters in the SMC. On the other hand, in Paper I we find that the LMC has $\sim 40 \%$ of very poor clusters. We also note that $90 \%$ of the clusters are either in the poor or very poor class. All these clusters have mass $<1700 M_{\odot}$. These suggest that the cluster population in the SMC is dominated by low mass clusters. This finding has implication to the cluster formation mechanism in the SMC.

\section{Results and discussion}

We have estimated ages and reddening of 179 star clusters in the SMC using a semi-automated quantitative method. Out of these, 17 clusters are parametrised for the first time. Out of 17 newly parameterised clusters, we find one (NGC 458) rich cluster and rest belong either to very poor or poor groups. We have also classified all the clusters based on their mass as well as strength for the first time. We have listed the results in a catalogue (available at the CDS). A sample of this catalogue is presented in Table 2. The catalogue contains the name of the clusters, position (RA and Dec as given in B08), radius, estimated ages and reddening by our method, previous estimation of ages by G10, PU99, C06, and group number based on our classification. In the catalogue, the clusters' name designated by an asterisk and blank spaces in Cols. 7 to 9 respectively imply newly parametrised clusters. There are three clusters (SK157, HW77 and HW82) in our catalogue with blank spaces in Cols. 7-9, whose ages are estimated by Piatti et al. (2015).

We have presented the field star decontaminated CMDs of all the 179 clusters, with over plotted M08 isochrones for the estimated age. Isochrones showing the typical uncertainty in the age estimation (0.25) are also over plotted. All the CMDs are presented in Appendix A. As an example, we have presented four CMDs from four groups $(I-I V)$ in Fig. 2. In the figure, cluster stars are denoted as black points, red solid line denotes the isochrone corresponding to estimated age and red dashed lines denote the isochrones corresponding to the age uncertainty. The turn-off of each isochrone is indicated with a red point. The name of the cluster and their corresponding age are mentioned on top of each subplot, along with their group number labelled in blue.

\subsection{Comparison with previous studies}

We found 119 clusters to be in common with G10, 56 clusters are in common with PU99 and 90 with C06. In Fig. 3 we have compared our age estimation ( $X$-axis) with previous estimations ( $Y$-axis). We have drawn a straight line with slope $=1$ in the plots to check the difference in age estimation. Clusters with different classification are denoted in different colours in the figure. The top left plot shows that our results match very well with G10 with an uncertainty of 0.25 in $\log$ scale except for a few very poor clusters. The top right plot also shows good matching of our results with PU99, although there are a few clusters for which we estimated older ages. Our results also match well within the error of $\log (t)=0.25$ with the estimation by C06 for most of the clusters older than $\sim 60 \mathrm{Myr}(\log (t)=7.8)$ (bottom panel). In the case of clusters younger than $60 \mathrm{Myr}$, we have estimated relatively older ages. We rechecked our CMDs of those 

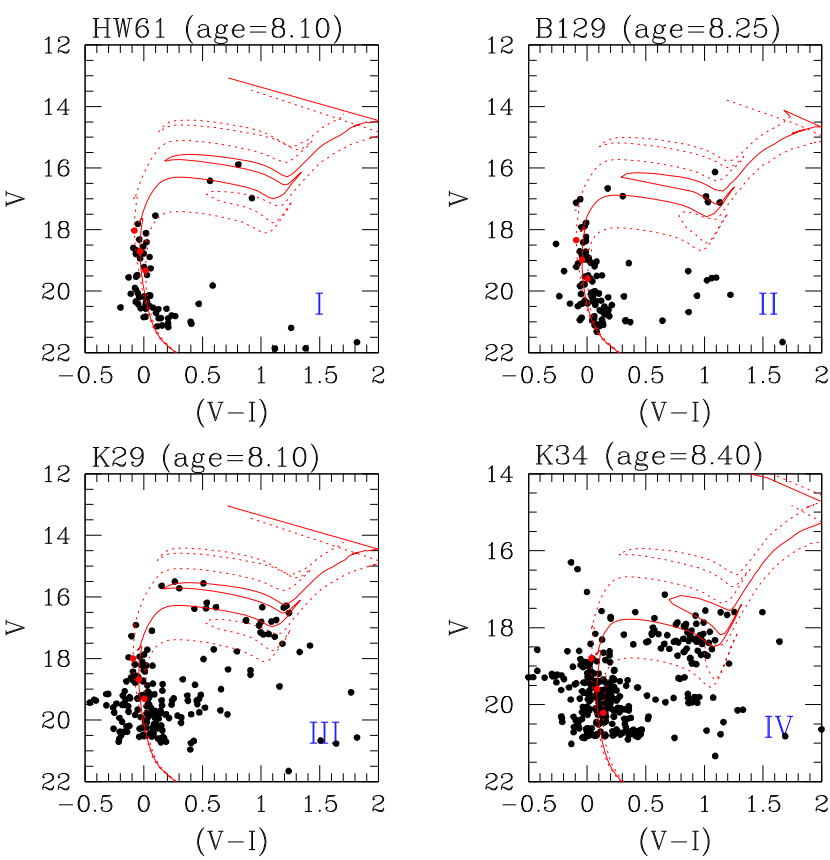

Fig. 2. CMDs of clusters from each group $(I-I V)$. The cluster's name and age $(\log (\mathrm{t}))$ are also marked.

clusters where we find a discrepancy in estimated ages with previous results and reconfirmed our estimated values.

In general, the comparison indicates that our estimations compare well with the previous studies. We also note a few cases of discrepancy, which could be due to reasons such as different data used by different authors, the difference in the isochrone models used, and the difference in adopted methods. PU99 and C06, used OGLE II data, which have lesser resolution than OGLE III data. Also, OGLE II covers the central region of the SMC, where the clusters may suffer more crowding effect. PU99 used isochrone model by Bertelli et al. (1994) and C06 used the isochrone model by Girardi et al. (2002), whereas G10 used MCPS data and two isochrone models for their analysis: Padova isochrones Girardi et al. (1995) and Geneva isochrones (Lejeune \& Schaerer 2001) for their age estimations.

\subsection{Reddening distribution}

We have constructed distribution of the estimated reddening $E(V-I)$ for different groups of clusters (shown in different colour) in Fig. 4. The distribution ranges from 0 to 0.4 mag for very poor and poor clusters, and from 0 to 0.3 mag for moderately rich clusters. The distribution peaks between 0.1 to 0.2 mag for all the four groups. We have compared the estimated reddening with the high resolution map of field reddening in the central SMC by Indu \& Subramaniam (2011) and found that they match well. We do not find any significant difference in reddening with that estimated using red clump stars by Subramanian \& Subramaniam (2012). We have also compared our estimated reddening with previous studies by G10, PU99 and C06. The distribution of difference is found to be peaked at $0.1 \mathrm{mag}$, which is within the error $(1 \sigma)$ of our estimation.

The spatial distribution of reddening across the SMC is plotted in Fig. 5. The red triangle indicates the centre of the SMC, located at $\left(0^{\mathrm{h}} 52^{\mathrm{m}} 45^{\mathrm{s}},-72^{\circ} 49^{\prime} 43^{\prime \prime}\right.$; Crowl et al. 2001). Most of the regions have reddening within $0.1-0.2 \mathrm{mag}$ with larger variation in reddening near the centre. The southern part of the SMC consists of clusters with relatively large reddening value than the north-eastern (NE) part.

\subsection{Strength distribution}

Spatial distribution of clusters as a function of their strength $\left(n_{\mathrm{m}}\right)$ is shown in Fig. 6. The red triangle indicates the centre of the SMC. The figure shows that clusters with $n_{\mathrm{m}}<100$ are distributed all over the SMC observed region. The clusters with $n_{\mathrm{m}}>100$ are preferentially located in the inner SMC, mainly close to the bar. We notice that there are two clusters with strength more than 200, located in the south-western (SW) and NE end of the SMC. We do not find any kind of hierarchical distribution of clusters in the SMC based on their strength, similar to that found in the LMC (Paper I). We notice the presence of only low mass clusters in the eastern SMC which is predominantly affected by the tidal forces (Besla et al. 2010, 2012).

\subsection{Age distribution}

Age distribution of various groups of clusters is shown in Fig. 7. We have used a bin width of 0.25 in log scale which is same as the error associated with the age estimation. The figure shows that very poor clusters are distributed over a large age range $(\log (t)=6.75-9.00)$ with peak at $\sim 130 \mathrm{Myr}(\log (t)=8.00-8.25)$. The poor clusters show two peaks: a younger peak at $130 \mathrm{Myr}$ $(\log (t)=8.00-8.25)$ and an older peak at $750 \mathrm{Myr}(\log (t)=$ 8.75-9.00). The moderately rich and rich clusters also show peak at $130 \mathrm{Myr}(\log (t)=8.00-8.25)$, similar to the very poor and poor clusters. Therefore, the clusters are mainly distributed between $30 \mathrm{Myr}$ to $300 \mathrm{Myr}(\log (t)=7.5-8.5)$. The cumulative distribution of all the studied clusters peaks at $\sim 130 \mathrm{Myr}$, which is almost same as that we identified in the LMC (Paper I). The fact that the cluster formation peaked at $\sim 130 \mathrm{Myr}$ in both the MCs could suggest that it is due a common triggering event. We suggest that the recent interaction ( 200-300 Myr ago) between the LMC and SMC might have triggered the cluster formation during the above mentioned age range. PU99 found the peak of cluster formation at around $30 \mathrm{Myr}$, whereas C06 found two peaks of cluster formation at $8 \mathrm{Myr}$ and $90 \mathrm{Myr}$. G10 found two peaks of cluster formation at 160 and $630 \mathrm{Myr}$. The younger peak shown in G10 is not very different from our younger peak.

The spatial distribution of age is shown in Fig. 8. The figure shows that clusters with age around $100 \mathrm{Myr}$ are distributed all over the SMC region. Western and south-eastern (SE) parts show older clusters. The central part consists of clusters with a relatively larger age range. The SMC stretches out from SW to $\mathrm{NE}$ direction and younger clusters are found in specific places, slightly north from the central region. Older clusters are mostly located in southern part of the SMC and northern part consists of relatively younger clusters. This is suggestive of preferential location of clusters as a function of age. This needs to be confirmed with a more complete sample of parameterised clusters.

We have therefore added 289 clusters from the previous studies which are not common to our catalogue. The clusters and their parameters are taken from G10, PU99, C06, (Piatti et al. 2005, 2007a, b, c, 2008, 2011, 2015), Piatti (2011, 2012), Maia et al. (2012), Mighell et al. (1998), Glatt et al. (2008), Girardi et al. (2013), Crowl et al. (2001), Parisi et al. (2014), Dias et al. (2014, 2016). As there are a large number of studies, we use a common reference, "other-studies", to indicate all the studies other than G10, PU99, and C06. The addition of clusters from previous studies not only increased the 

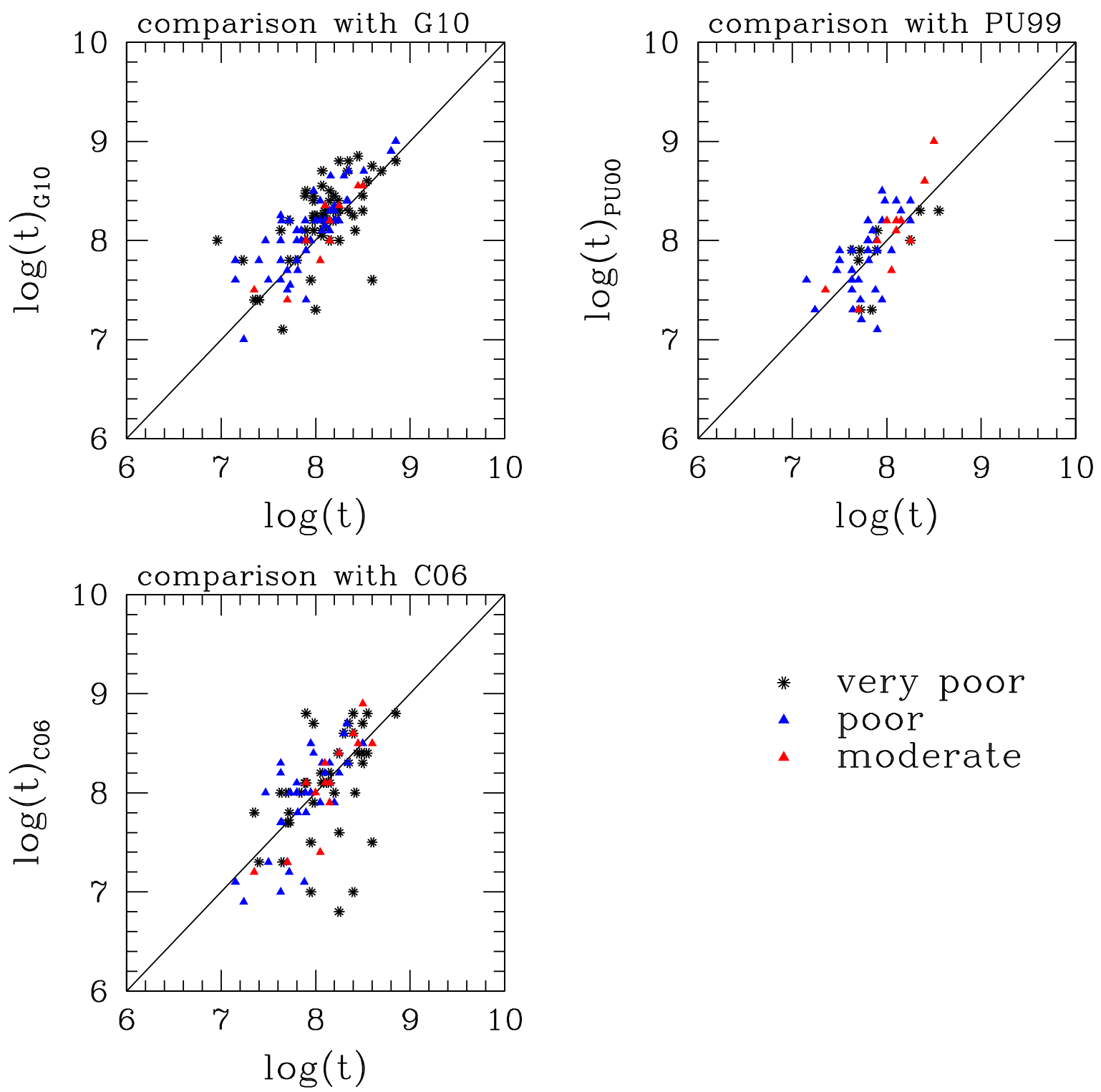

Fig. 3. Comparison between our estimated age ( $X$-axis) and age estimated by previous studies ( $Y$-axis). We have compared our age estimation with that of G10 (top left), PU99 (top right) and C06 (bottom one). Different point types indicate clusters from different group of classification. A straight line with slope $=1$ is shown in each plot to indicate the deviation in the estimated age.

cluster sample but also the coverage of the SMC. The total sample of 468 clusters is large enough to study the spatio-temporal distribution of clusters, which is discussed in the next section. This is the largest parameterised sample of clusters in the SMC. The age distribution of this compiled sample of 468 clusters is also shown in Fig. 7. The distribution shows two peaks (130 and $750 \mathrm{Myr}$ ) of cluster formation. The younger peak is found to be same as that estimated from our sample.

Spatial distribution of the compiled sample as a function of age is shown in Fig. 9. The centre of the SMC is denoted by solid red square. The distribution suggests that older clusters are mostly located at the southern and western part of the SMC, whereas, the younger clusters are found in the inner SMC along with a few clusters in the east. The clusters with ages $\sim 100 \mathrm{Myr}$ are distributed all over the SMC. The distribution also suggests that the SMC is stretched out from SW to NE along the bar, could be due to the interaction between the MCs. We find that the north-west quadrant of the Fig. 9 is devoid of clusters. We suggest that the reasons could be either due to lack of available photometric data in that region resulting in no parameterised clusters, or due to genuine lack of clusters in this part of the SMC. It is important to fill this gap in the spatial distribution of SMC clusters. A genuine lack of clusters in this region could put constraints on the cluster formation as well as gas distribution in the SMC.

\subsection{Spatio-temporal distribution}

To understand the spatio-temporal distribution, the spatial location of clusters in various age range is shown in Fig. 10. The ages of the clusters are in the range of $\log (t)=6.8-9.1$. The black points in the figure denote the clusters from our catalogue, the red small circles are the clusters from G10, the blue points are from C06 and PU99, and the clusters from "other-studies" are denoted as cyan points. The green point indicates the centre of the SMC. As shown in Fig. 10d, clusters in the age range $630 \mathrm{Myr}-1.25 \mathrm{Gyr}$ are mostly found in the southern and western parts of the SMC including the central region. Very few clusters are found in the northern and eastern regions during 


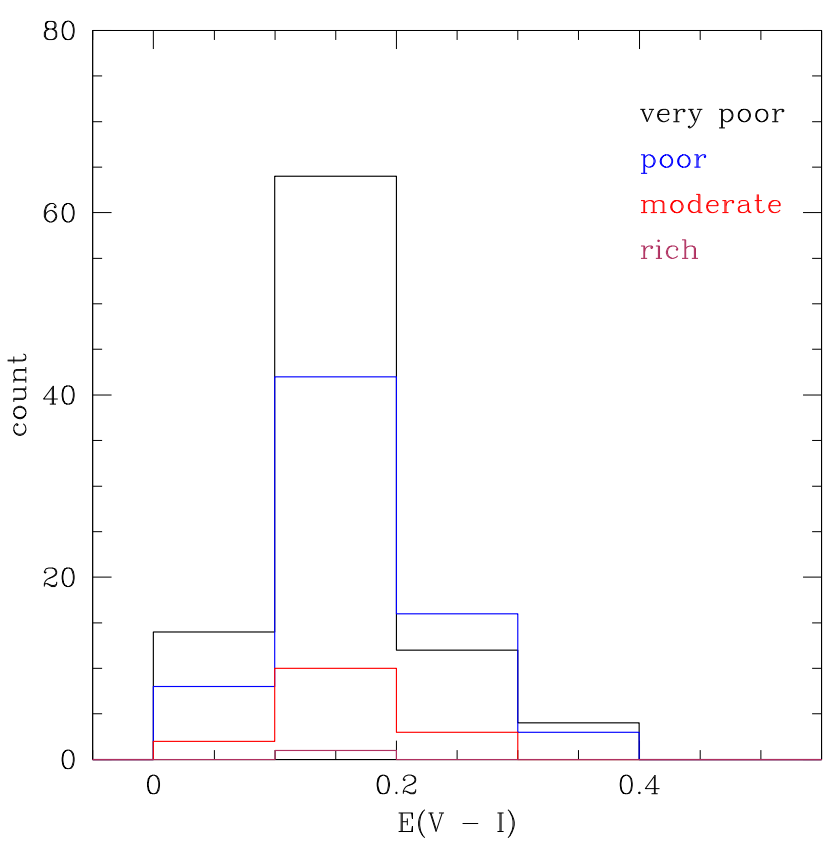

Fig. 4. Reddening distribution of very poor (black), poor (blue), moderate (red) and rich (maroon) clusters. Reddening value peaks between 0.1 and 0.2 mag for all the groups.

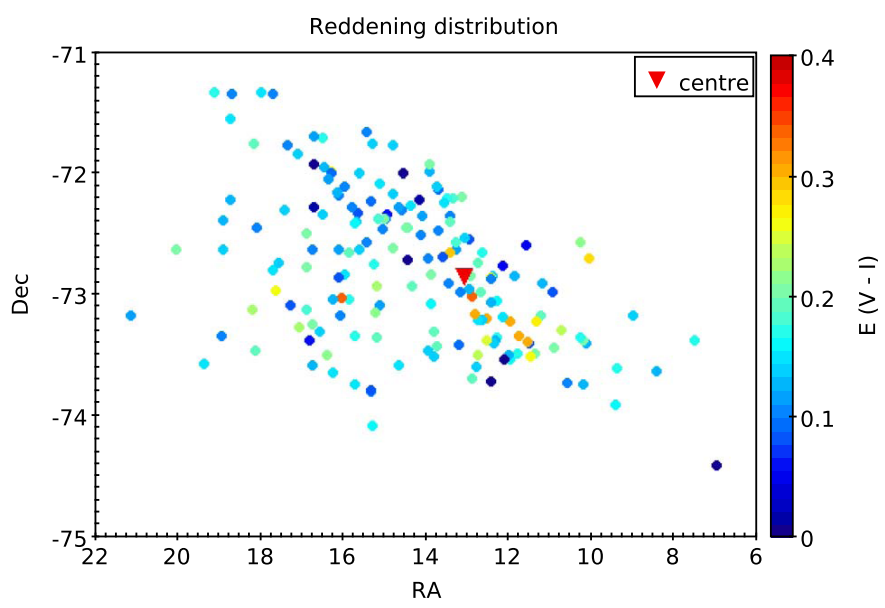

Fig. 5. Spatial variation of estimated reddening across the SMC.

this period. During the period 250-630 Myr (Fig. 10c), the clusters are found mostly in the central region, along with a group of clusters in the NE region. On the other hand, the western and the southern regions are devoid of clusters.

In the age range 100-250 Myr (Fig. 10b), most of the clusters are found in the eastern and NE regions along with the central SMC. The western and southern regions continue to be devoid of clusters during this period. We also find that the extent of the NE region is maximum during this period. Figure 10a shows the location of clusters formed in the last $100 \mathrm{Myr}$. These are found to be mostly in the NE region and the central SMC. We notice a specific pattern in the distribution of clusters; different from the other three panels.

Figure 10a and d shows the distribution of clusters in two extreme epochs. The spatial distribution can be seen to be distinctly different with no co-relation between the two epochs. Most of the clusters in the older epoch are in the southern part, whereas the ones in the younger epoch are mostly found in the northern and central region. Figure $10 \mathrm{~b}$ and $\mathrm{c}$ shows the shift of clusters from

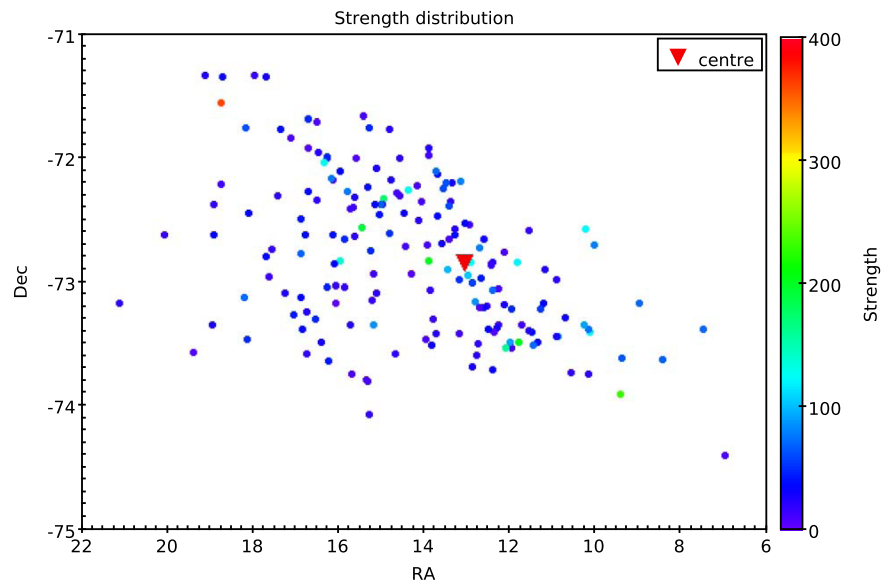

Fig. 6. Spatial distribution of star clusters in the SMC as a function of cluster strength.

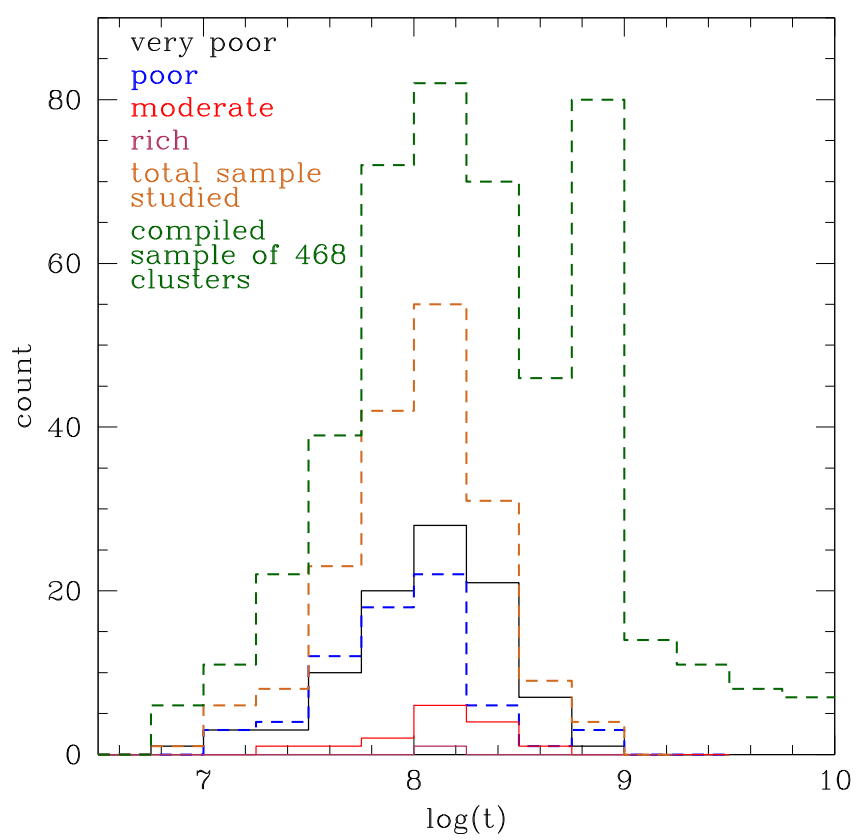

Fig. 7. Age distribution of very poor (black), poor (blue), moderate (red), and rich (maroon) clusters. Distributions of all the four groups of clusters peak in the range of $\log (t)=8.00-8.25$. Cumulative age distribution of all the studied clusters (chocolate) shows a peak at $\sim 130 \pm 35$ Myr. Age distribution of the compiled sample of 468 clusters peaks at $\sim 130$ and $\sim 750 \mathrm{Myr}$.

south to north. Similar shift was found in star formation during the same period by Harris \& Zaritsky (2004, their Fig. 6). Figure 10 also suggests that the central region of the SMC is actively forming clusters from $\sim 1 \mathrm{Gyr}$ till date. We suggest that a close interaction between the LMC and the SMC 1.2 Gyr ago (Diaz \& Bekki 2011) may be the reason for triggering cluster formation in the southern and the western part of the SMC (Fig. 10d). We also suggest that the recent interaction at $250 \mathrm{Myr}$ caused cluster formation in the last $100 \mathrm{Myr}$, resulting in the spatial distribution as shown in Fig. 10a. Figure 13 of Besla et al. (2012) showed that the SMC made close passages around the LMC at $\sim 900 \mathrm{Myr}$ and $100 \mathrm{Myr}$ ago, which supports the above observation. The spatial distribution of clusters presented in Fig. 10 could give important clues regarding the details of the interactions.

We made two videos (available online) to understand the spatio-temporal distribution in detail. In the video-1, we have 


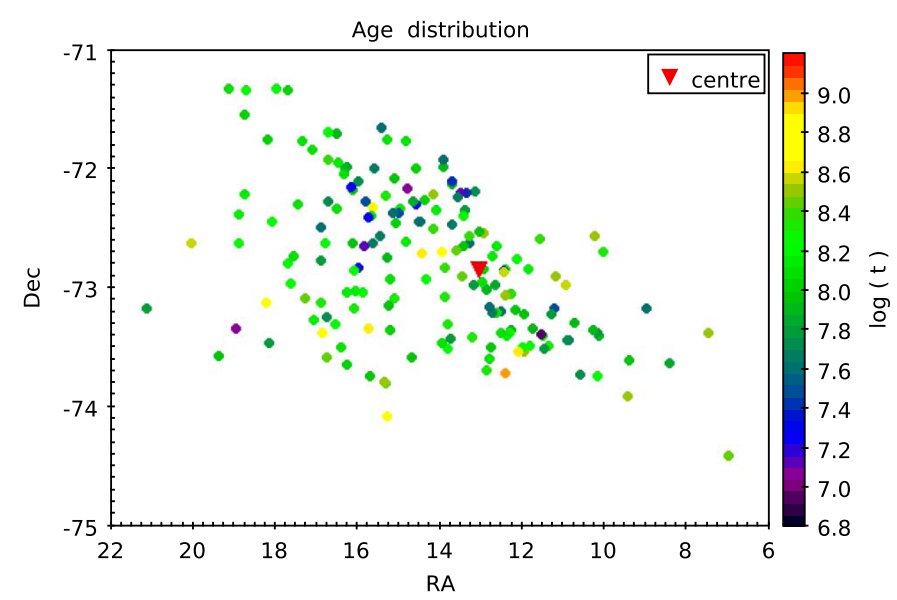

Fig. 8. Spatial distribution of star clusters in the SMC as a function of age.

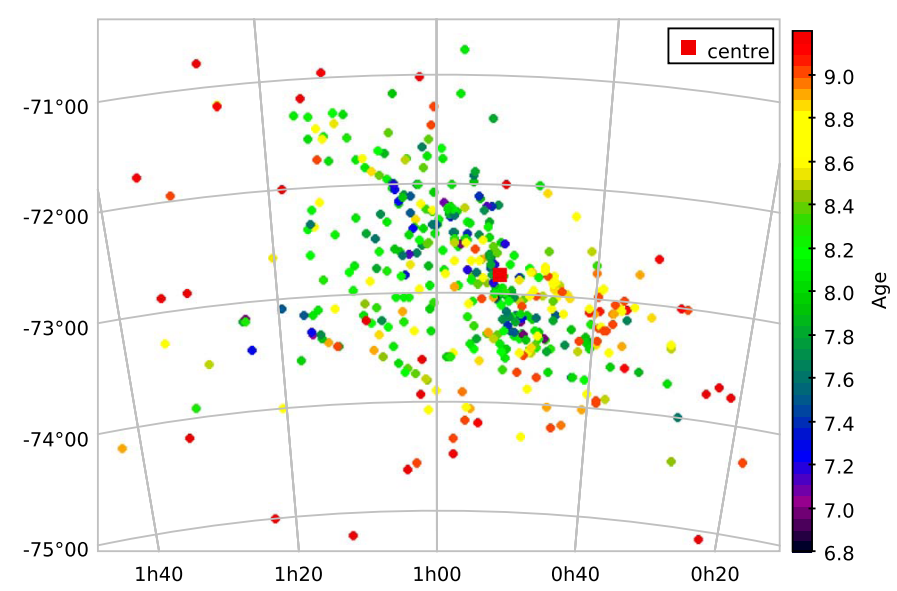

Fig. 9. Spatial distribution of the compiled sample of 468 star clusters in the SMC as a function of age.

shown the cluster distribution from older to younger age, and video- 2 shows vice-versa. In the videos, we have used the same colour notations as in Fig. 10. The two videos clearly demonstrate the change in location of clusters as a function of age. The details of spatio-temporal distribution of this largest cluster sample will provide important details of cluster formation history in the SMC. The distribution shown in Fig. 10 are in fact snapshots from the videos for specific epochs. Many such snapshots can be created for various epochs as required using these videos.

In the case of the LMC (Paper I), we identified an outside to inside propagation of cluster formation. On the other hand, in the SMC, we identify a progressive shifting of cluster location from the south to the north during the last $600 \mathrm{Myr}$. The clusters older than 1.25 Gyr are found to be distributed in the outskirts of the SMC. We identified both the MCs to have a peak in cluster formation at $\sim 130 \mathrm{Myr}$. This is suggestive of a common cluster formation trigger, which is most likely to be the recent interaction between the MCs. The details of spatio-temporal distribution of clusters presented in this study together with Paper I can be used as a tool to constrain details of the recent LMC-SMC interactions.

\subsection{Mass-radius relation}

In order to understand the structure of the SMC clusters, we have plotted radius of clusters $(\log (r))$ against the strength $\left(\log \left(n_{m}\right)\right)$ for our studied sample (filled circle) in Fig. 11. There are five clusters with $n_{m}>400$ (categorised as rich clusters), which we excluded for parameterisation, are also shown in this figure. To compare the structure of the SMC clusters with that of the LMC, we have over plotted the LMC clusters data (open box), taken from Paper I. Clusters with different classification are denoted in different colour. The figure shows that there is linear relation between radius and strength in logarithmic scale for both the MCs. The figure also suggests that though there is a spread in the radius of clusters with similar strength for both the MCs, the SMC clusters tend to have systematically larger radii than LMC clusters with similar strength. This points to the possibility of compactness of the LMC clusters when compared to the SMC clusters. In order to shed more light on this, it is necessary to estimate the nature of relation between cluster mass and radius.

Figure 12 shows the relation between radius $(\log (r))$ and mass $(\log \mathrm{M})$ of clusters in the SMC. We took an average of mass range of clusters and average radius of clusters for different groups $(I-V)$ to estimate the co-relation between them. The data points of the LMC and SMC are marked as blue and black respectively. Straight lines fitted to the data points give slopes of $2.10( \pm 0.24)$ and $1.68( \pm 0.43)$ for the LMC and the SMC respectively. The difference in slope is of the order of $1-\sigma$, hence the result is only indicative. There is an indication that the clusters with similar mass occupy smaller radius in the case of the LMC than in the SMC, indicating that the SMC clusters are loosely bound when compared to those in the LMC. Pfalzner et al. (2016) derive a similar relation for star clusters in the solar neighbourhood using the relation $\mathrm{M}_{c}=\mathrm{C}_{m} \times R^{\gamma}$. They find the value of $\gamma$ as $1.7 \pm 0.2$ for a large range of cluster mass. They also mention that it is necessary to find out if there exists a universal relation between mass and radius of clusters. We find that the values of $\gamma$ are similar for the SMC and the Galaxy, whereas it is marginally higher for the LMC. This suggests that cluster formation environment in the SMC is similar to that of our Galaxy in the solar neighbourhood. Our study shows that there is a tentative evidence for tighter clusters in the LMC, when compared to those in the Galaxy and the SMC. This needs to be verified with the help of better data including individual mass and radii estimates of clusters.

\section{Summary}

We have estimated the age and reddening of 179 star clusters in the SMC using OGLE III data and presented a catalog (available at the CDS). Out of 179, 17 clusters are parameterised for the first time. Out of 17 newly parameterised clusters, one is rich (NGC 458) while the rest belong to very poor or poor group. Field star decontaminated CMDs of all the 179 clusters, fitted with isochrones of estimated age and corrected for reddening are presented in the appendix. The SMC star clusters are classified based on their mass and richness in four groups for the first time. It has been found that $90 \%$ of our studied sample has mass $<1700 M_{\odot}$, which suggests that the SMC is dominated by low mass clusters. The lower mass limit of the SMC star clusters is found to be very similar to that of the open clusters in the Galaxy. We have also found a tentative evidence for tighter clusters in the LMC, when compared to the LMC and our Galaxy.

Combining our sample with previous studies, we compiled age information of 468 clusters to study their spatio-temporal distribution. The age distribution in the SMC peaks at $130 \pm$ $35 \mathrm{Myr}$, similar to the LMC (Paper I). We suggest that this could be due to the most recent LMC-SMC interaction. The clusters with age $630 \mathrm{Myr}-1.25 \mathrm{Gyr}$ are found to be located 
P. K. Nayak et al.: Age-dating, classification, and spatio-temporal distribution of the SMC clusters
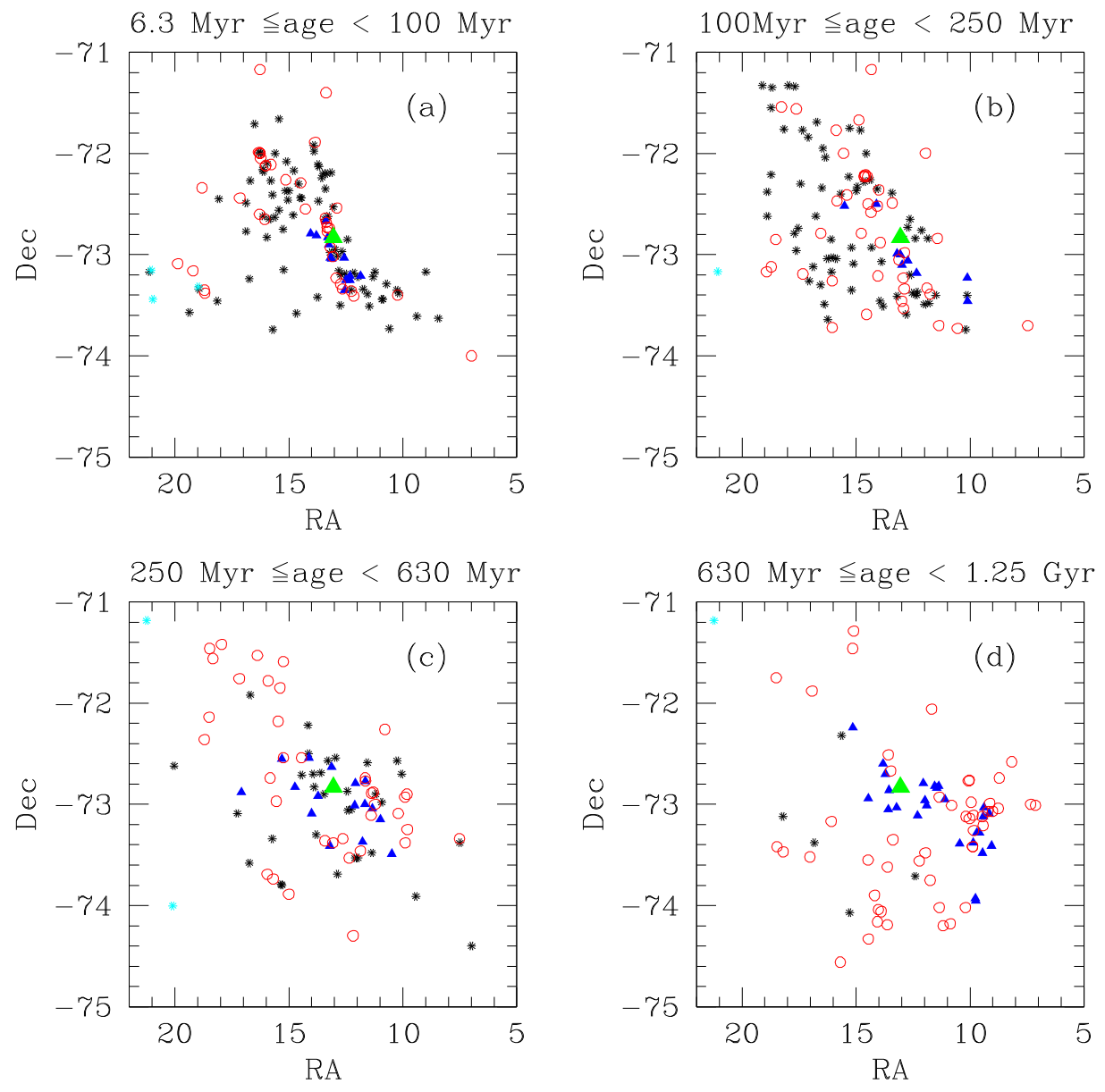

Fig. 10. Spatial location of the SMC clusters at different epochs in each panel, where the clusters are taken from our catalogue (black), by G10 (red circle), C06 and PU99 (blue), "other-studies" (cyan).

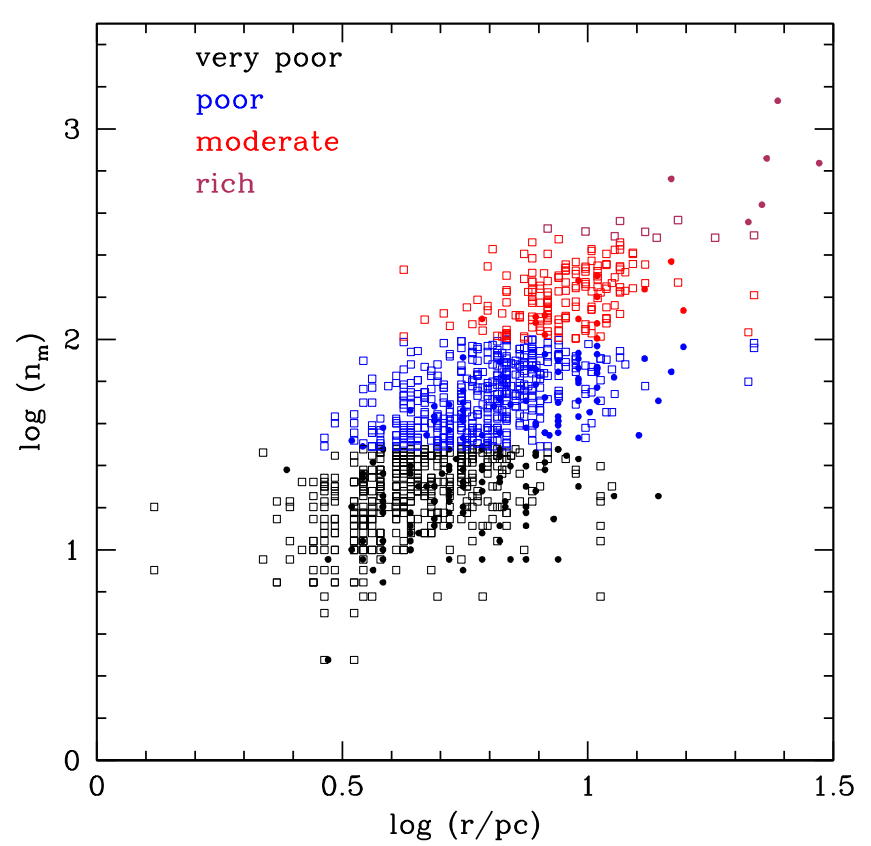

Fig. 11. Relation between radius and strength of clusters in the LMC (open box) and the SMC (filled circle). Clusters of different classification are denoted by different colours.

preferentially in the South and West of the SMC, whereas the clusters younger than $100 \mathrm{Myr}$ are found in the North and eastern regions, suggesting a shift in the location of cluster formation. The central SMC shows a continuous formation of clusters in

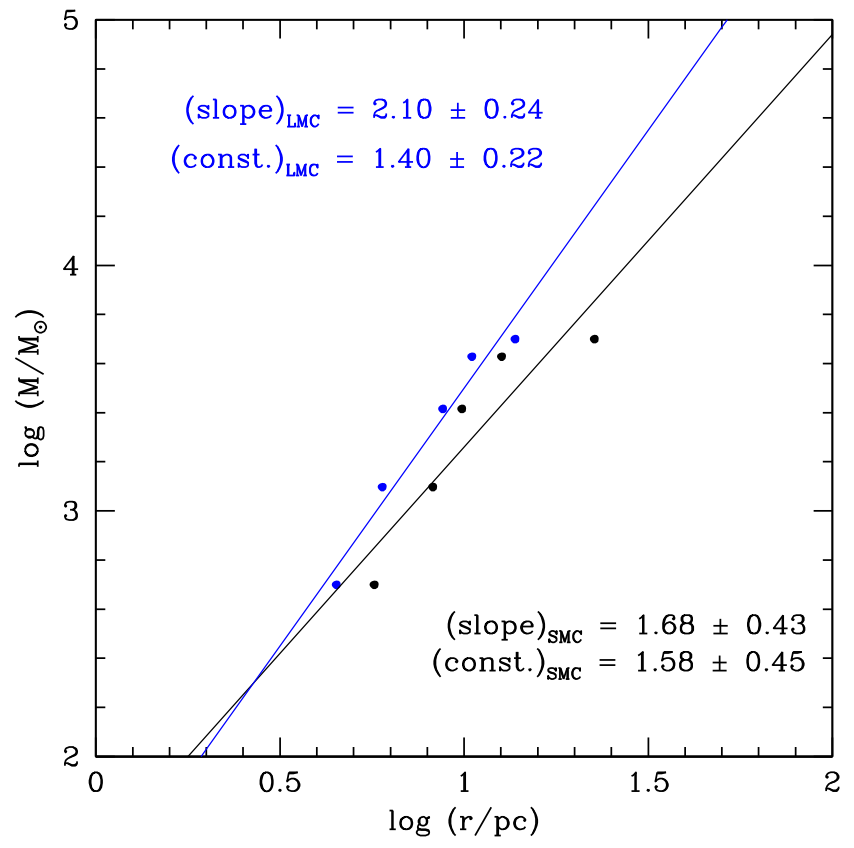

Fig. 12. Relation between average radius and average mass of clusters of different groups. The blue data points correspond to the LMC and the black ones correspond to the SMC. The slope and $y$-intercept of linear fit are also mentioned in the figure.

the last 1 Gyr. The details of this spatio-temporal shift is presented in two videos (available online). The details of spatiotemporal distribution of clusters presented in this study together 
with Paper I can be used as a tool to constrain details of the recent LMC-SMC interactions.

Acknowledgements. Ram Sagar would like to acknowledge the award of NASISenior Scientist Platinum Jubilee Fellowship by the National Academy of Science, Allahabad, India. S. Choudhury would like to thank the support from Basic Science Research Program through the National Research Foundation of Korea (NRF) funded by the Ministry of Education (NRF2016R1D1A1B01006608) and that by the KASI-Yonsei Joint Research Program for all Frontiers of As tronomy and Space Science funded by the Korea Astronomy and Space Science Institute. P. K. Nayak would like to thank Dr. Avijeet Prasad (Udaipur Solar Observatory, Physical Research Laboratory, India) for helping with Mathematica code to make the videos. The authors thank the OGLE team for making the data available in public domain.

\section{References}

Belokurov, V., Erkal, D., Deason, A. J., et al. 2017, MNRAS, 466, 4711

Bertelli, G., Bressan, A., Chiosi, C., Fagotto, F., \& Nasi, E. 1994, A\&AS, 106, 275

Besla, G., Kallivayalil, N., Hernquist, L., et al. 2010, ApJ, 721, L97

Besla, G., Kallivayalil, N., Hernquist, L., et al. 2012, MNRAS, 421, 2109

Bica, E., Bonatto, C., Dutra, C. M., \& Santos, J. F. C. 2008, MNRAS, 389, 678

Chiosi, E., Vallenari, A., Held, E. V., Rizzi, L., \& Moretti, A. 2006, A\&A, 452 179

Crowl, H. H., Sarajedini, A., Piatti, A. E., et al. 2001, AJ, 122, 220

Deason, A. J., Belokurov, V., Erkal, D., Koposov, S. E., \& Mackey, D. 2017, MNRAS, 467, 2636

Dias, B., Kerber, L. O., Barbuy, B., et al. 2014, A\&A, 561, A106

Dias, B., Kerber, L., Barbuy, B., Bica, E., \& Ortolani, S. 2016, A\&A, 591, A11

Diaz, J., \& Bekki, K. 2011, MNRAS, 413, 2015

Diaz, J. D., \& Bekki, K. 2012, ApJ, 750, 36

Girardi, L., Chiosi, C., Bertelli, G., \& Bressan, A. 1995, A\&A, 298, 87

Girardi, L., Bertelli, G., Bressan, A., et al. 2002, A\&A, 391, 195

Girardi, L., Goudfrooij, P., Kalirai, J. S., et al. 2013, MNRAS, 431, 3501

Glatt, K., Grebel, E. K., Sabbi, E., et al. 2008, AJ, 136, 1703

Glatt, K., Grebel, E. K. \& Koch, A. 2010, A\&A, 517, A50

Harris, J., \& Zaritsky, D. 2004, AJ, 127, 1531

Harris, J., \& Zaritsky, D. 2009, AJ, 138, 1243

Hunter, D. A., Elmegreen, B. G., Dupuy, T. J., \& Mortonson, M. 2003, AJ, 126, 1836

Indu, G., \& Subramaniam, A. 2011, A\&A, 535, A115

Kallivayalil, N., van der Marel, R. P., Alcock, C., et al. 2006, ApJ, 638, 772

Kallivayalil, N., van der Marel, R. P., Besla, G., Anderson, J., \& Alcock, C. 2013 ApJ, 764, 161

King, I. 1962, AJ, 67, 471

Kontizas, M., Danezis, E., \& Kontizas, E. 1982, A\&AS, 49, 1

Lamers, H. J. G. L. M., Gieles, M., Bastian, N., et al. 2005, A\&A, 441, 117
Lejeune, T., \& Schaerer, D. 2001, A\&A, 366, 538

Mackey, A. D., \& Gilmore, G. F. 2003, MNRAS, 338, 120

Maia, F., Piatti, A. E., \& Santos, Jr., J. F. C. 2012, Boletín de la Asociación Argentina de Astronomía La Plata Argentina, 55, 111

Maia, F. F. S., Piatti, A. E., \& Santos, J. F. C. 2014, MNRAS, 437, 2005

Marigo, P., Girardi, L., Bressan, A., et al. 2008, A\&A, 482, 883

Mathewson, D. S., Cleary, M. N., \& Murray, J. D. 1974, ApJ, 190, 291

Mighell, K. J., Sarajedini, A., \& French, R. S. 1998, AJ, 116, 2395

Nayak, P. K., Subramaniam, A., Choudhury, S., Indu, G., \& Sagar, R. 2016, MNRAS, 463, 1446

Nidever, D. L., Majewski, S. R., Butler Burton, W., \& Nigra, L. 2010, ApJ, 723, 1618

Nikolaev, S., Drake, A. J., Keller, S. C., et al. 2004, ApJ, 601, 260

Parisi, M. C., Grocholski, A. J., Geisler, D., Sarajedini, A., \& Clariá, J. J. 2009, AJ, 138, 517

Parisi, M. C., Geisler, D., Carraro, G., et al. 2014, AJ, 147, 71

Pfalzner, S., Kirk, H., Sills, A., et al. 2016, A\&A, 586, A68

Piatti, A. E. 2011, MNRAS, 416, L89

Piatti, A. E. 2012, ApJ, 756, L32

Piatti, A. E., \& Bica, E. 2012, MNRAS, 425, 3085

Piatti, A. E., Sarajedini, A., Geisler, D., Seguel, J., \& Clark, D. 2005, MNRAS, 358, 1215

Piatti, A. E., Sarajedini, A., Geisler, D., Clark, D., \& Seguel, J. 2007a, MNRAS, 377,300

Piatti, A. E., Sarajedini, A., Geisler, D., Gallart, C., \& Wischnjewsky, M. 2007b, MNRAS, 382, 1203

Piatti, A. E., Sarajedini, A., Geisler, D., Gallart, C., \& Wischnjewsky, M. 2007c, MNRAS, 381, L84

Piatti, A. E., Geisler, D., Sarajedini, A., Gallart, C., \& Wischnjewsky, M. 2008, MNRAS, 389, 429

Piatti, A. E., Clariá, J. J., Bica, E., et al. 2011, MNRAS, 417, 1559

Piatti, A. E., de Grijs, R., Rubele, S., et al. 2015, MNRAS, 450, 552

Pietrzynski, G., \& Udalski, A. 1999, Acta Astron., 49, 157

Piskunov, A. E., Kharchenko, N. V., Schilbach, E., et al. 2008, A\&A, 487, 557

Putman, M. E., Staveley-Smith, L., Freeman, K. C., Gibson, B. K., \& Barnes, D. G. 2003, ApJ, 586, 170

Ruprecht, J. 1966, Bull. Astr. Inst. Czechosl, 17, 33

Salpeter, E. E. 1955, ApJ, 121, 161

Searle, L., Wilkinson, A., \& Bagnuolo, W. G. 1980, ApJ, 239, 803

Storm, J., Carney, B. W., Gieren, W. P., et al. 2004, A\&A, 415, 531

Subramanian, S., \& Subramaniam, A. 2009, A\&A, 496, 399

Subramanian, S., \& Subramaniam, A. 2012, ApJ, 744, 128

Trumpler, R. J. 1930, Lick Observatory Bulletin, 14, 154

Udalski, A. 2003, Acta Astron., 53, 291

Udalski, A., Szymanski, M., Kubiak, M., et al. 1998, VizieR Online Data Catalog: J/AcA/48/147

Udalski, A., Soszyński, I., Szymański, M. K., et al. 2008, Acta Astron., 58, 329

Wannier, P., \& Wrixon, G. T. 1972, ApJ, 173, L119

Zaritsky, D., Harris, J., Thompson, I. B., Grebel, E. K., \& Massey, P. 2002, AJ, 123,855

Zaritsky, D., Harris, J., Thompson, I. B., \& Grebel, E. K. 2004, AJ, 128, 1606 


\section{Appendix A: CMDs of 179 catalogued clusters}

Field star decontaminated CMDs of all the catalogued clusters are presented in this section. M08 isochrones for estimated age and typical uncertainty (0.25) associated with it are over plotted on each cluster CMD. The cluster stars are presented as black points, whereas red solid line and dashed line represents the age and its uncertainty respectively. The turn-off point of each isochrone is denoted by red point. The name of the cluster and their corresponding age are mentioned on top of each CMD. The clusters' name designated by an asterisk imply newly parametrised clusters. This is same as Fig. 2.

For a few clusters, we have used three field regions to decontaminate the cluster CMD, and over-plotted three decontaminated CMDs to identify cluster sequence. Cluster stars corresponding to three different decontamination processes are denoted as black points, blue crosses and blue circles.
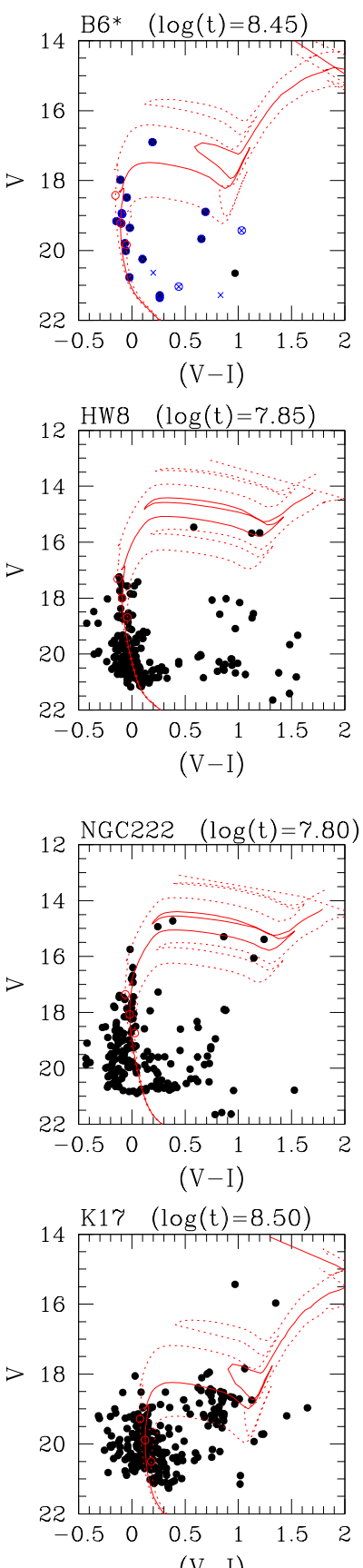

$(\mathrm{V}-\mathrm{I})$
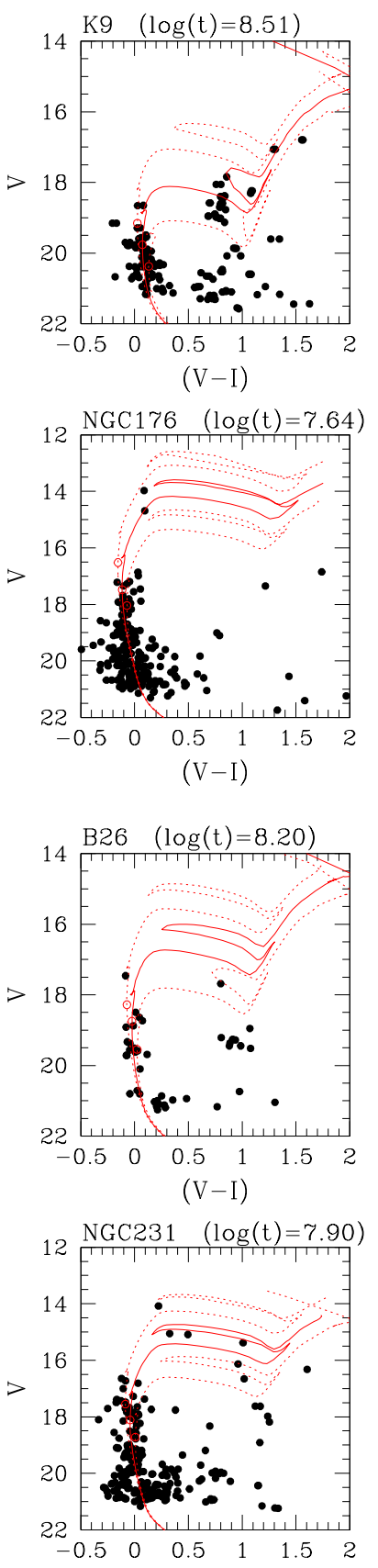

$(\mathrm{V}-\mathrm{I})$
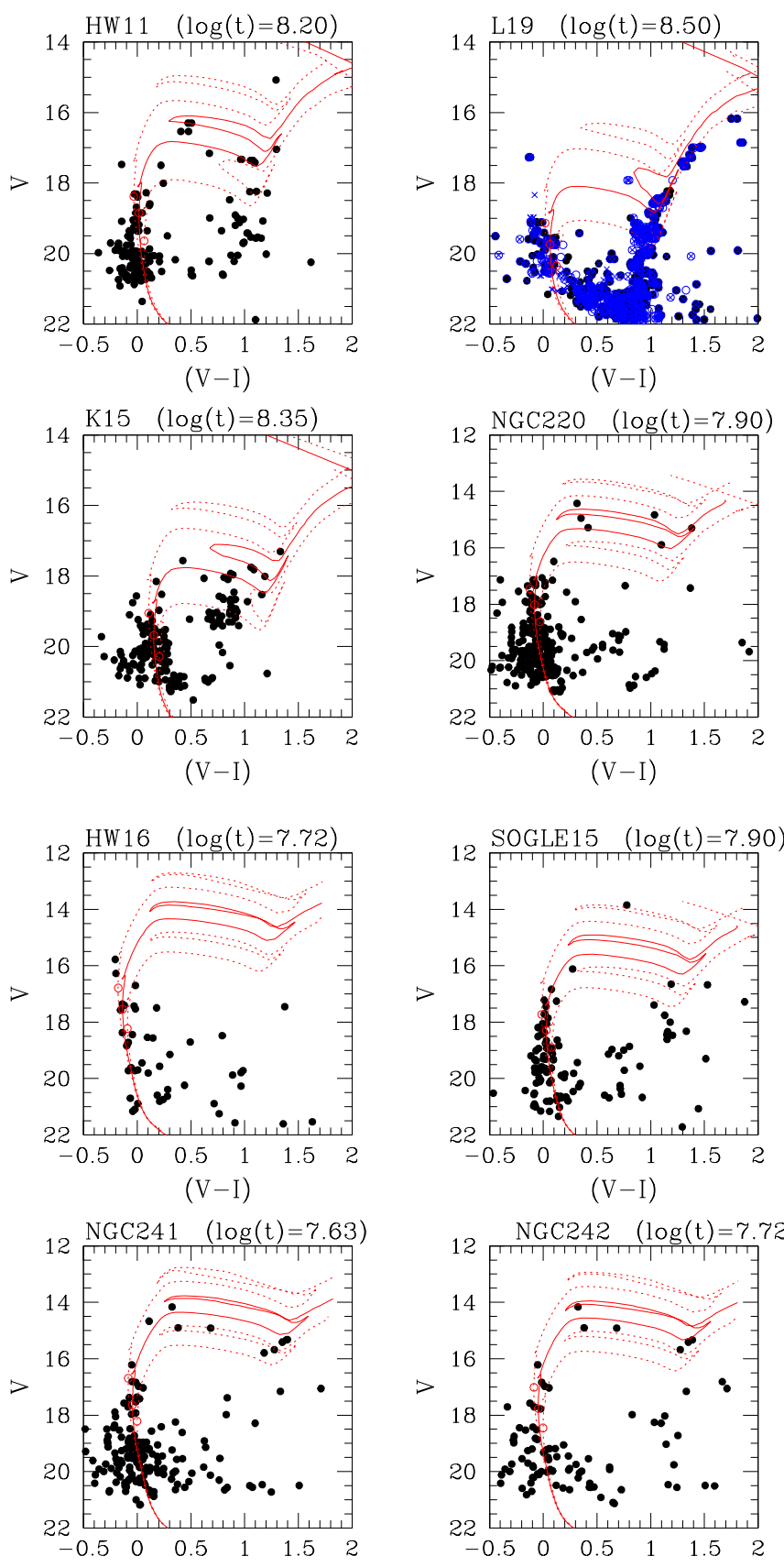

$(\mathrm{V}-\mathrm{I})$

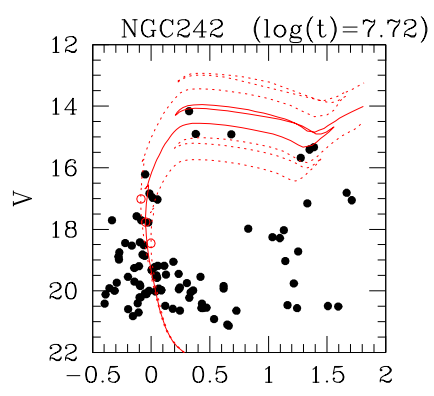

$(\mathrm{V}-\mathrm{I})$

Fig. A.1. Each panel represents the de-contaminated CMD of a catalogued cluster with estimated age. Colours and symbols used are explained in the text of appendix. 

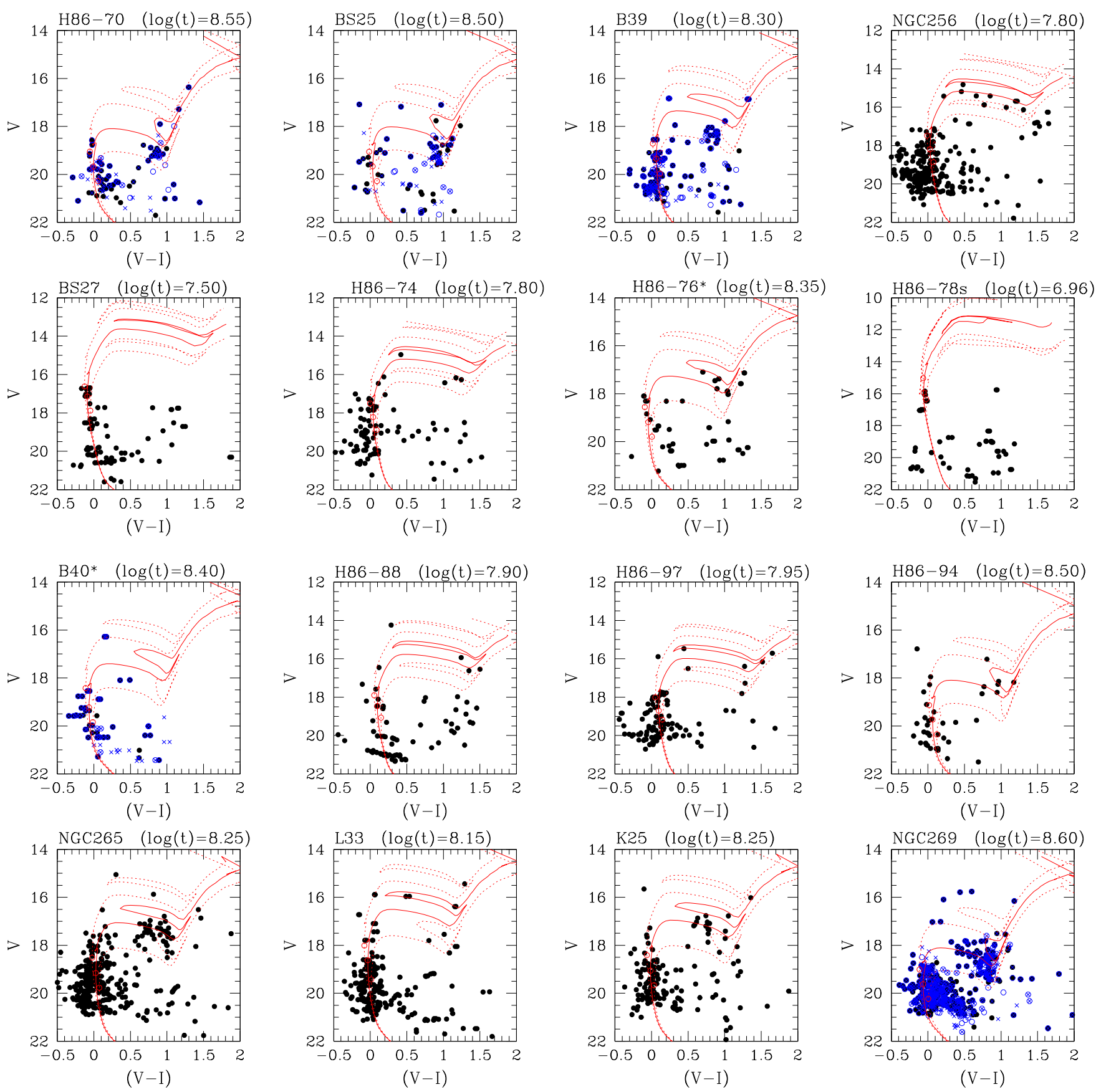

Fig. A.1. continued. 
P. K. Nayak et al.: Age-dating, classification, and spatio-temporal distribution of the SMC clusters
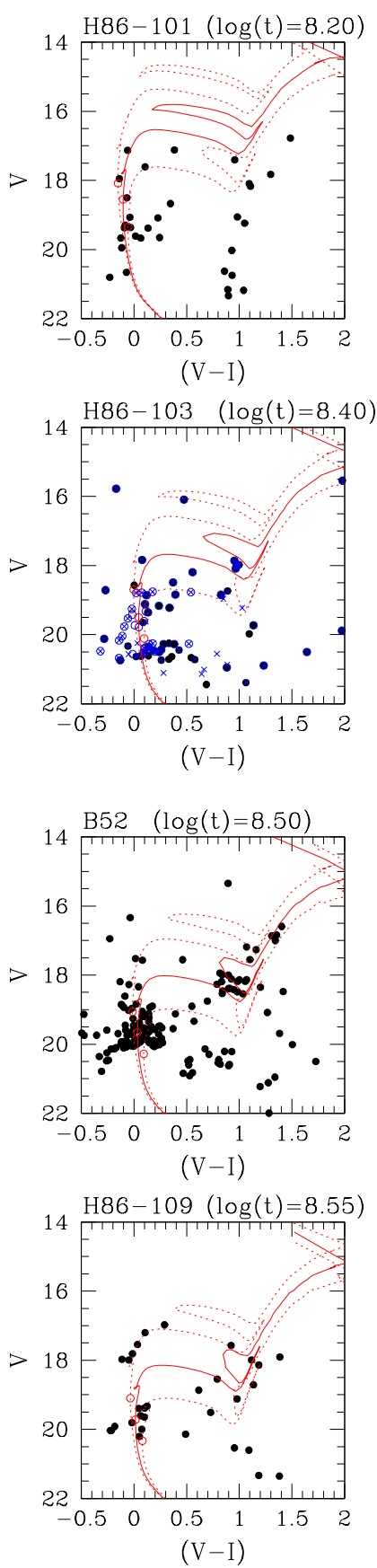

Fig. A.1. continued.
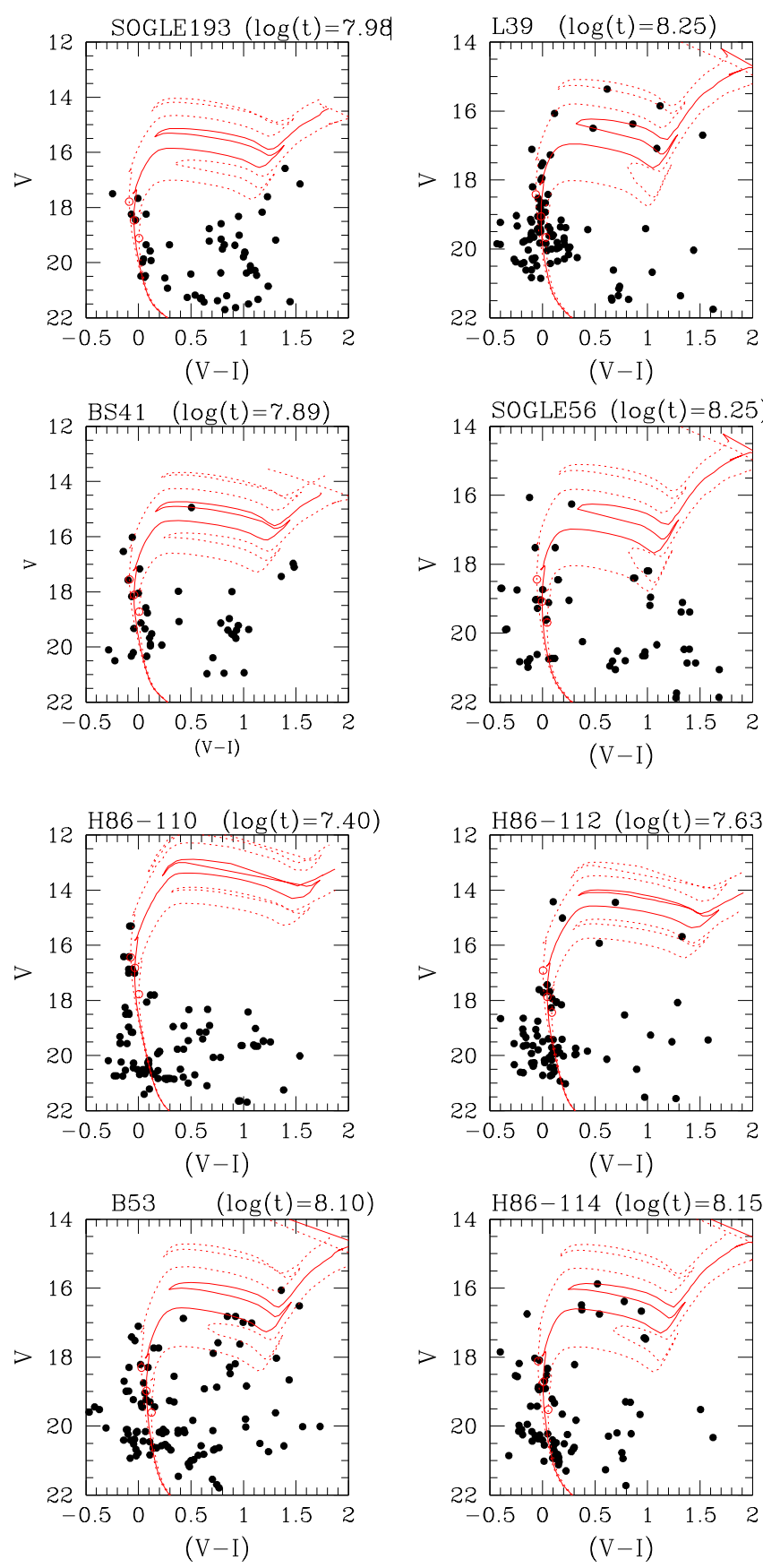
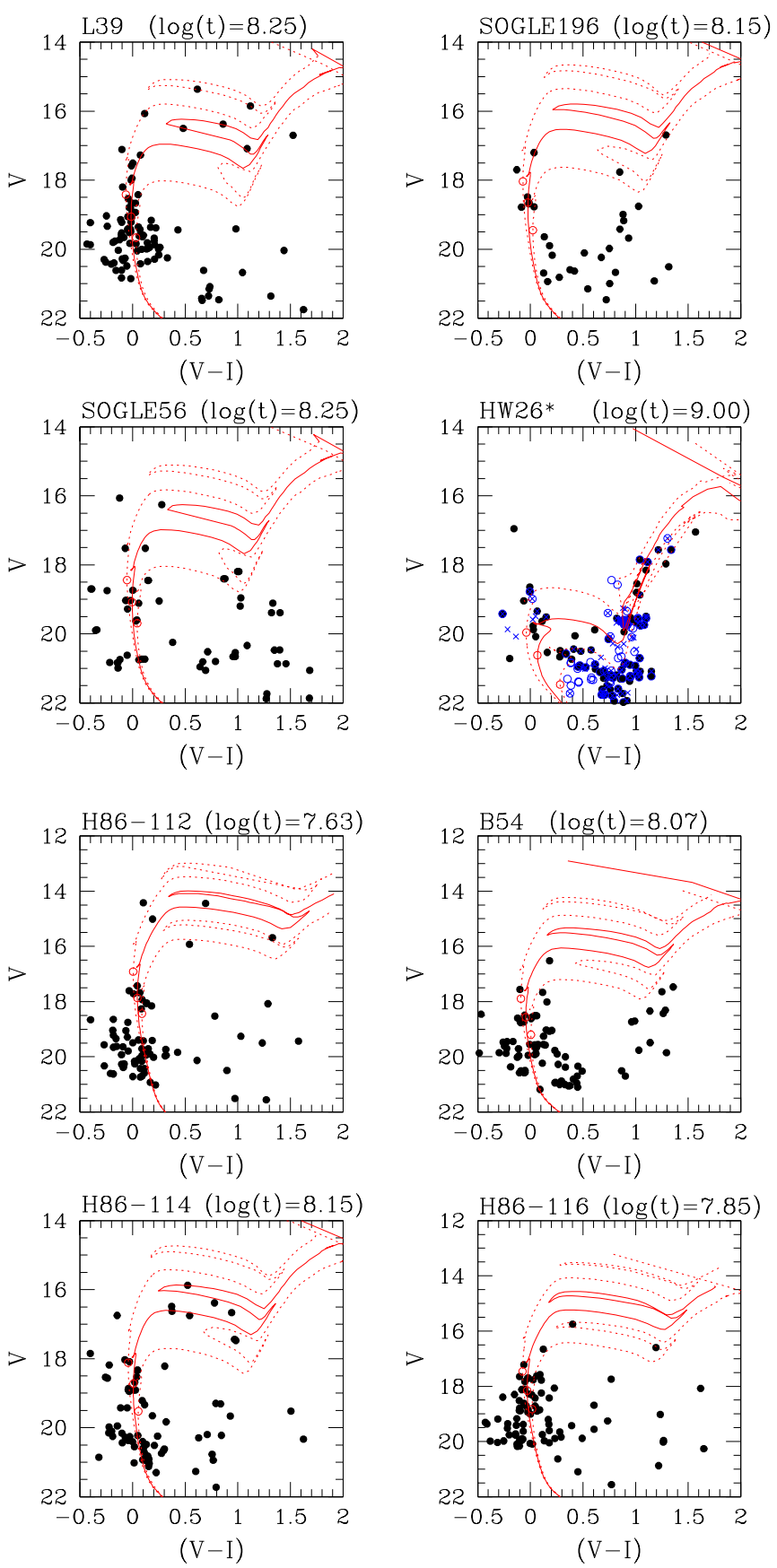

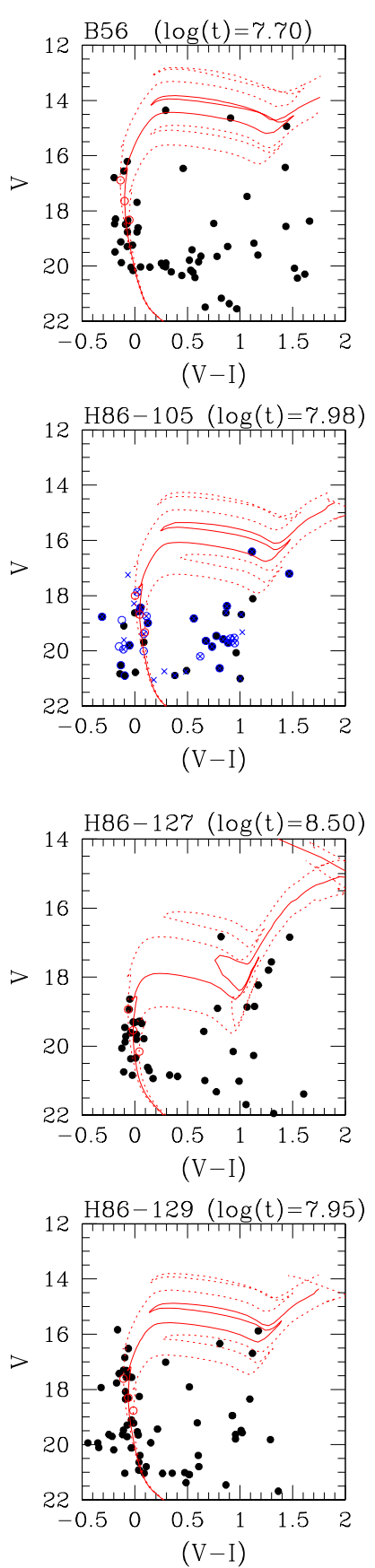

Fig. A.1. continued.
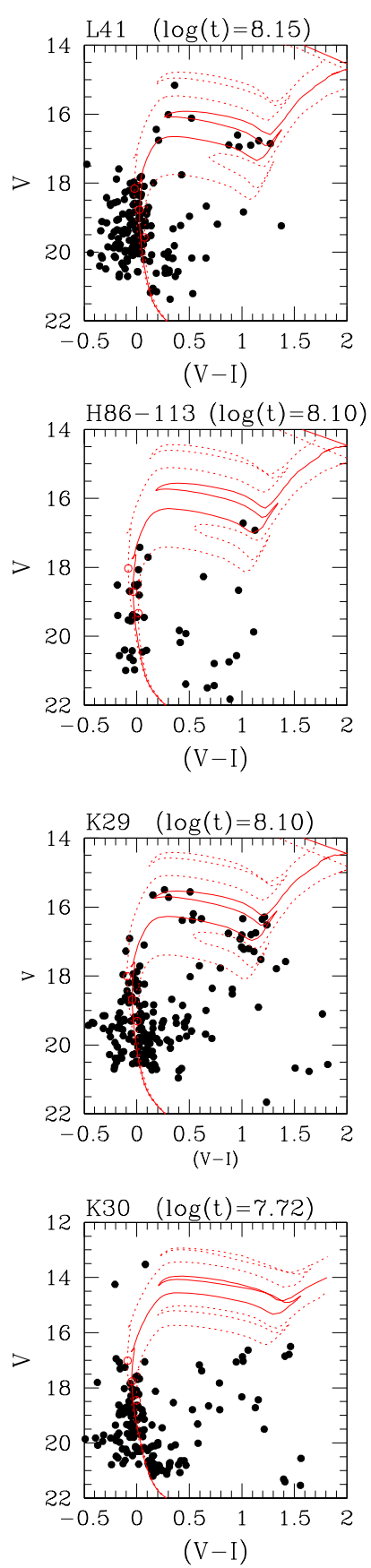
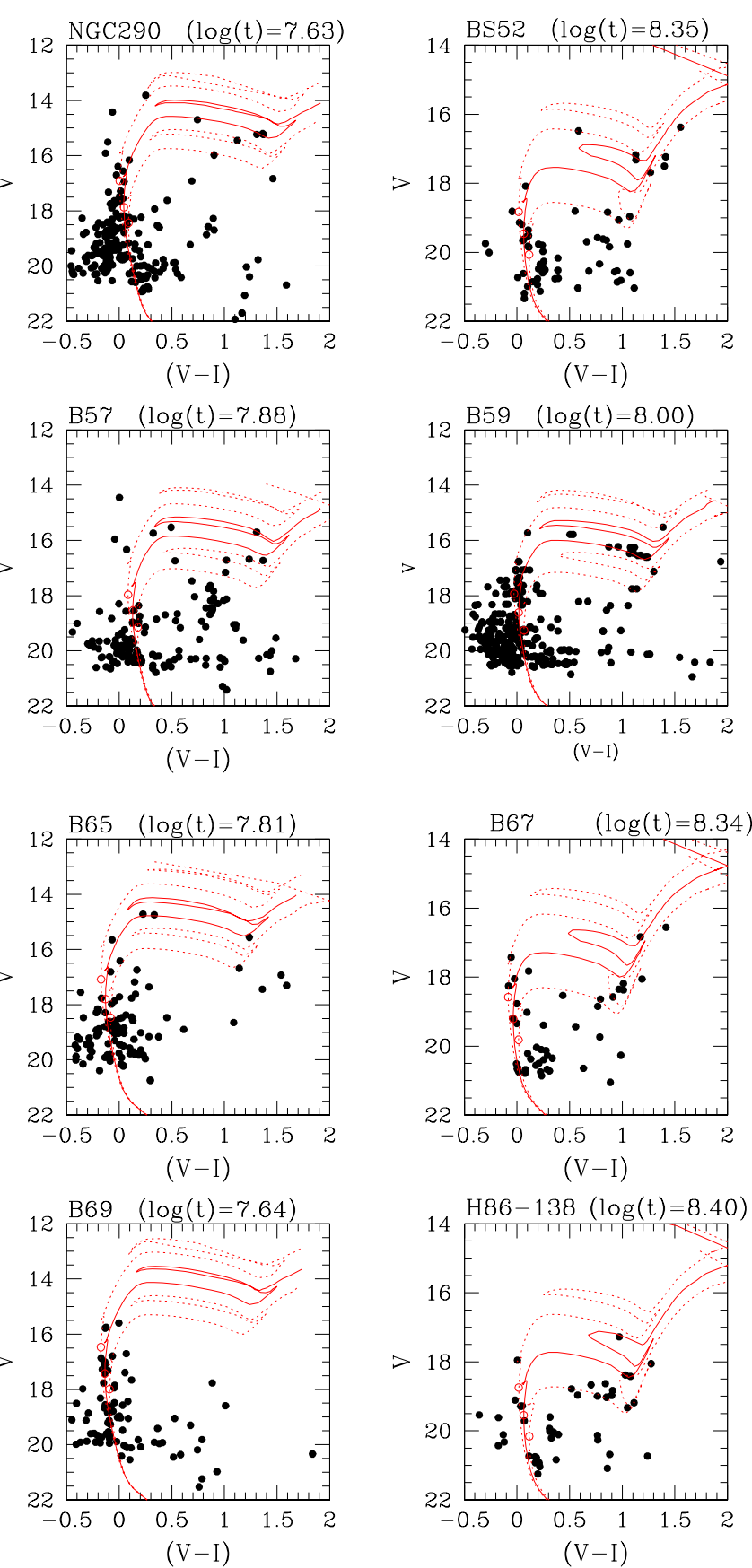
P. K. Nayak et al.: Age-dating, classification, and spatio-temporal distribution of the SMC clusters
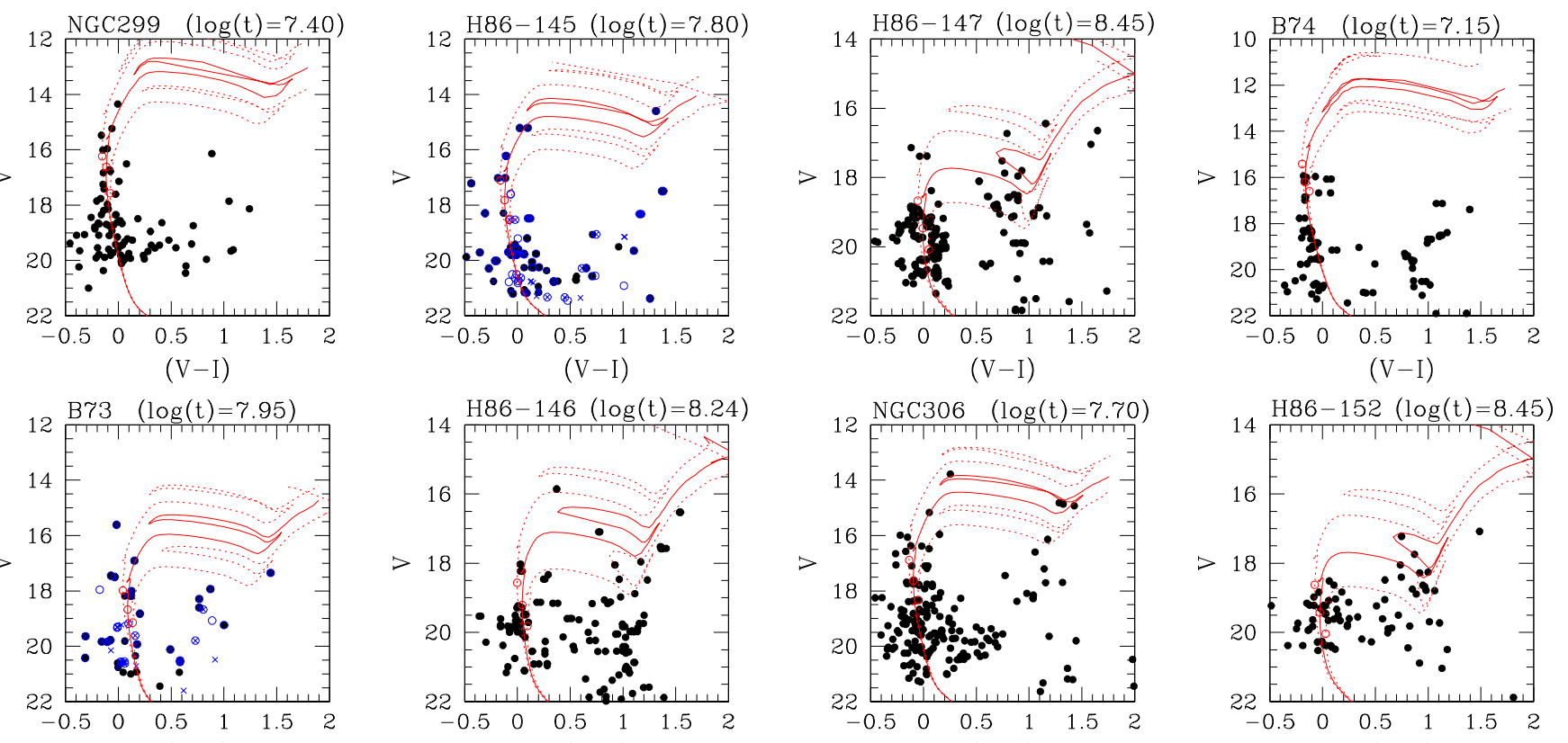

$(\mathrm{V}-\mathrm{I})$

$(\mathrm{V}-\mathrm{I})$
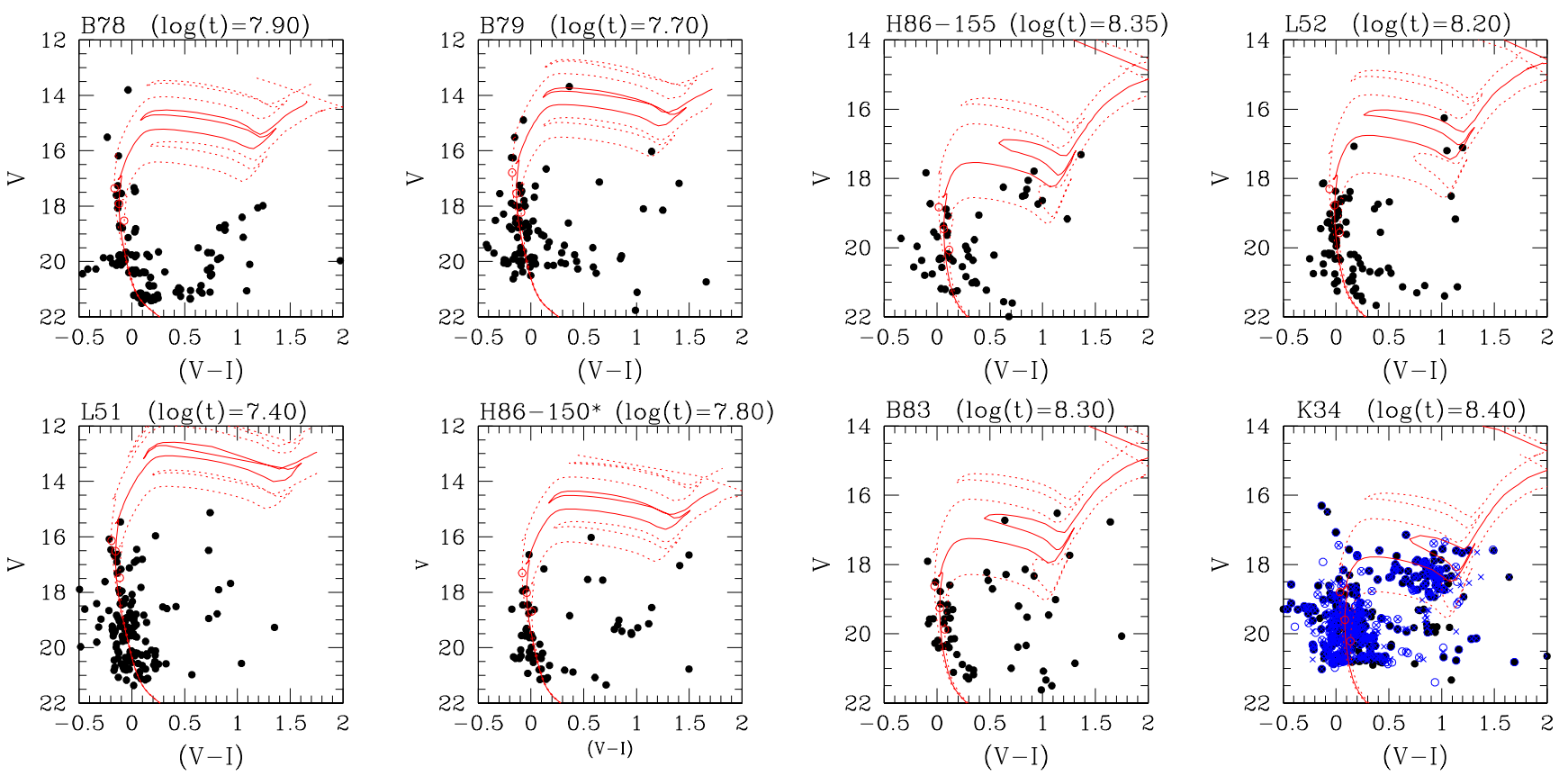

Fig. A.1. continued. 

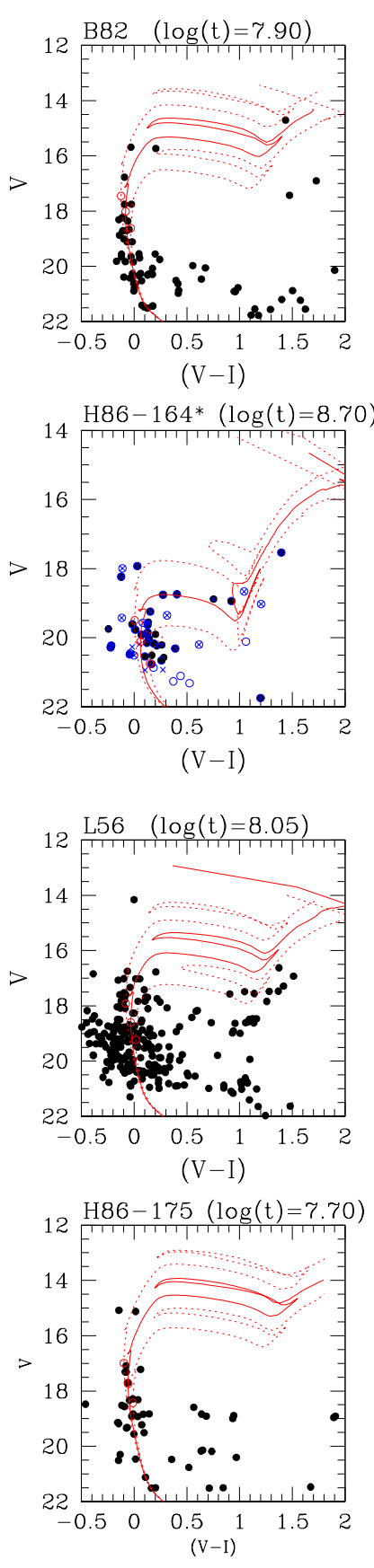

Fig. A.1. continued.
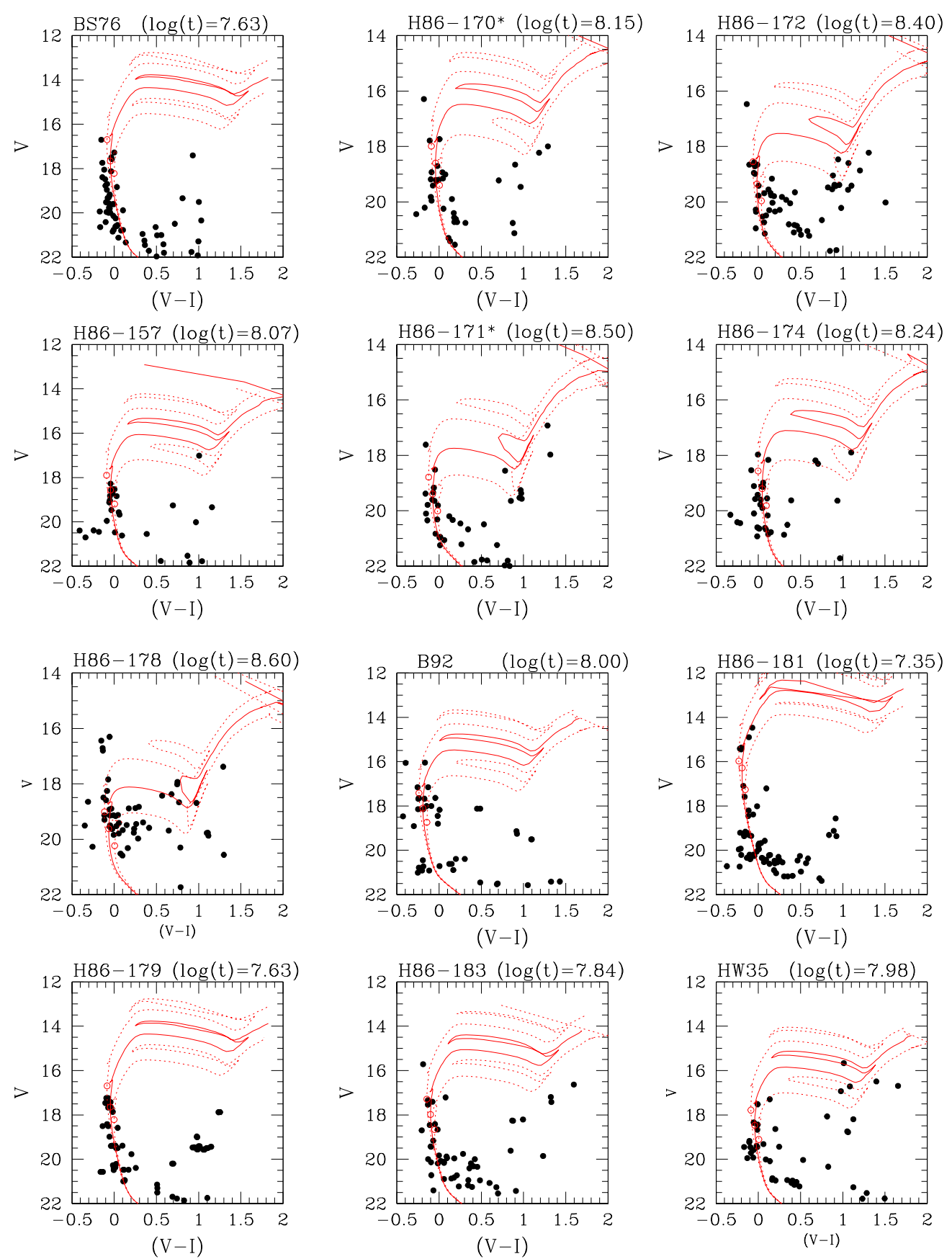
P. K. Nayak et al.: Age-dating, classification, and spatio-temporal distribution of the SMC clusters
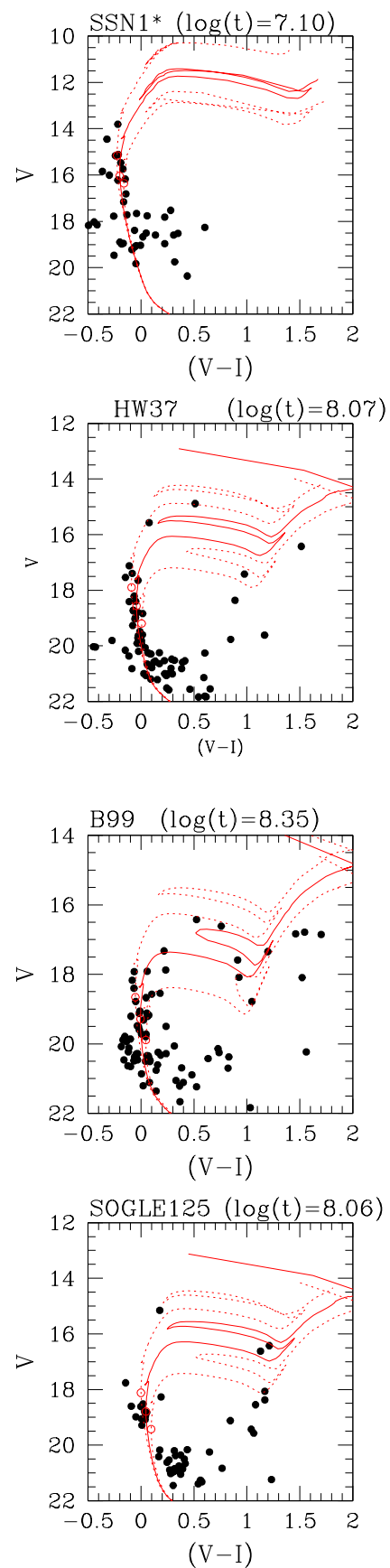
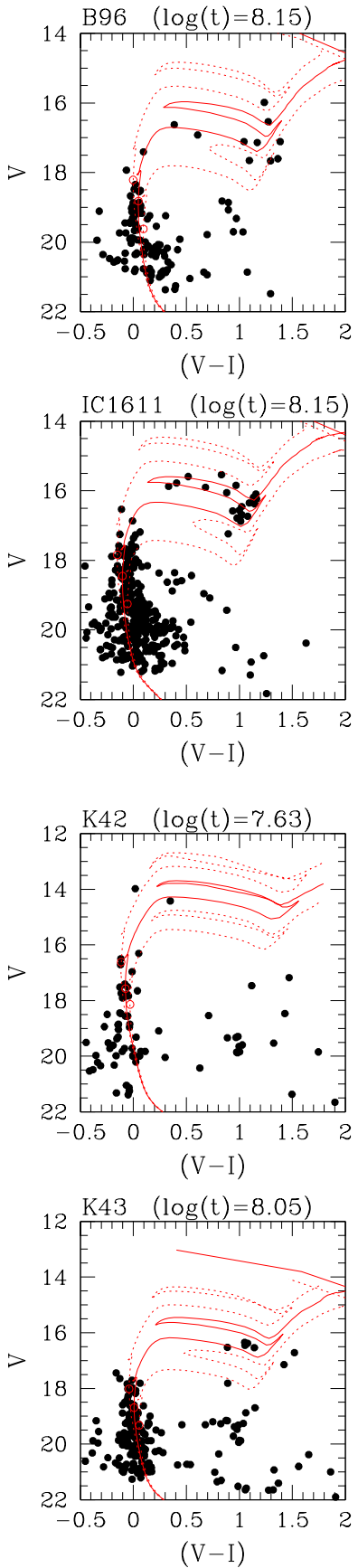

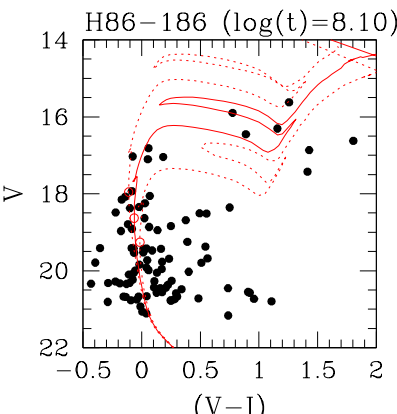

$(\mathrm{V}-\mathrm{I})$
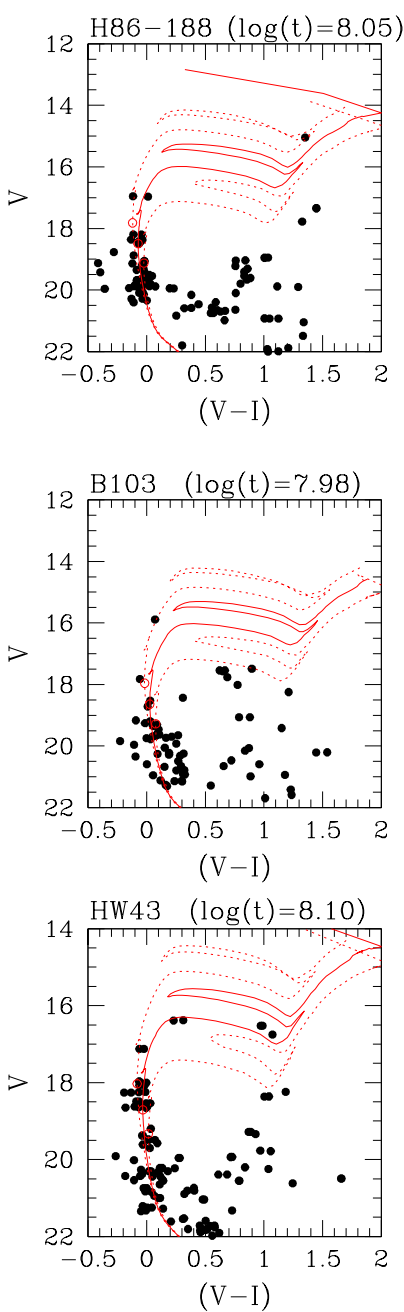
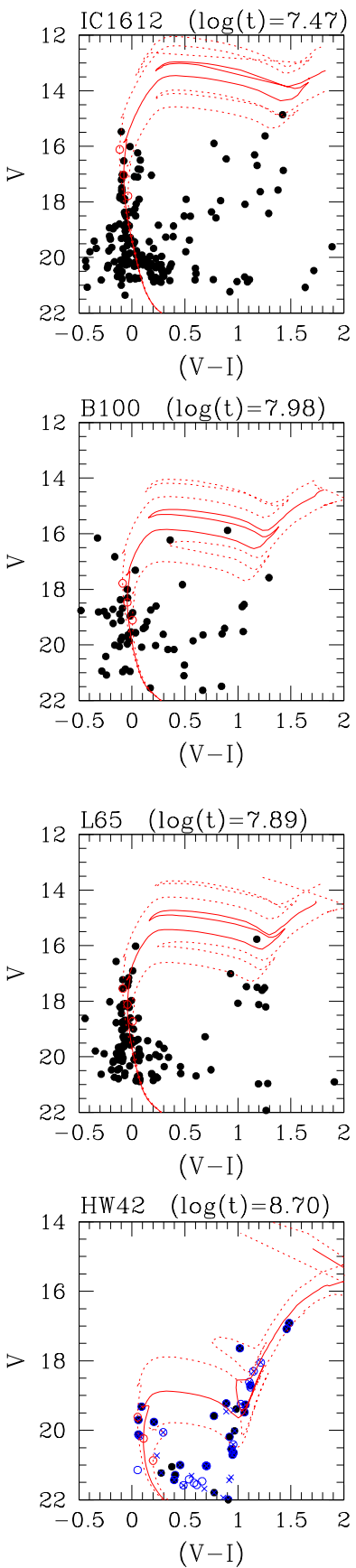

Fig. A.1. continued. 

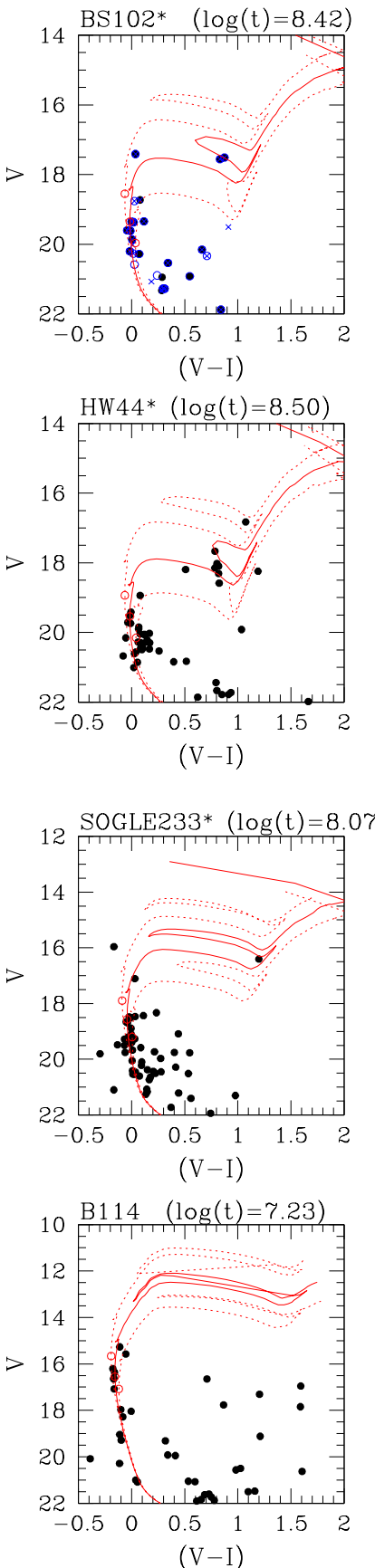

Fig. A.1. continued.
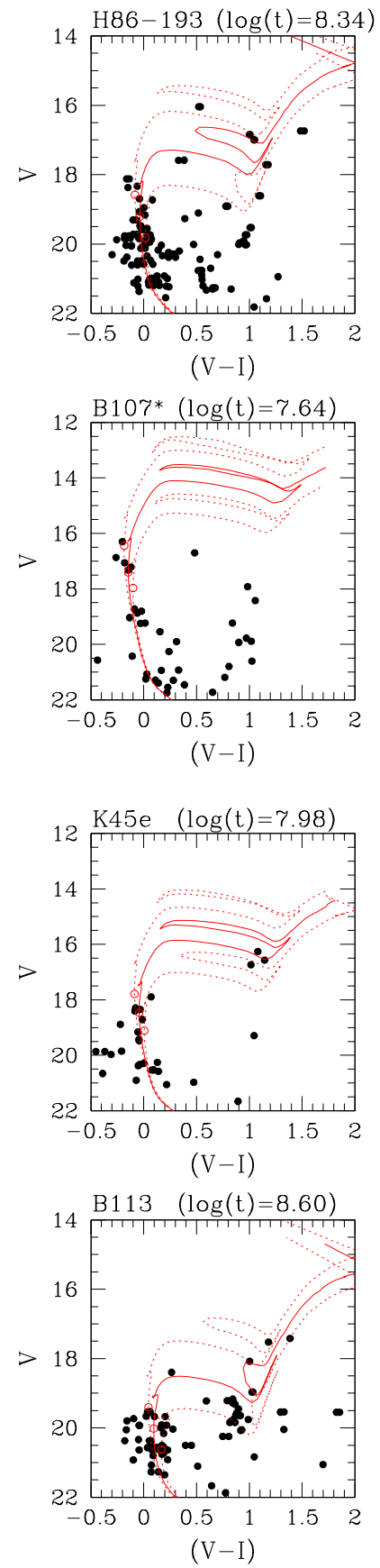
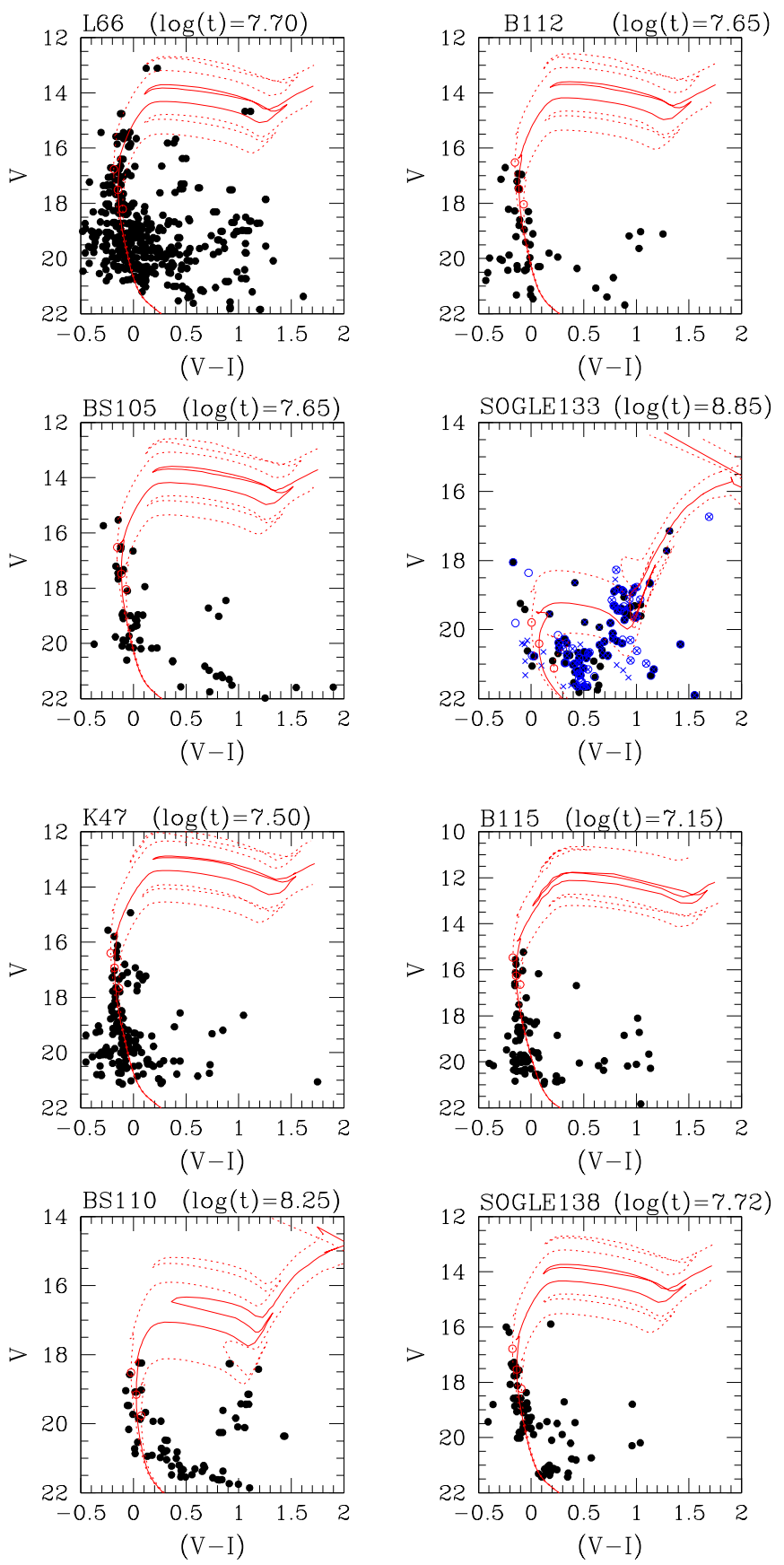
P. K. Nayak et al.: Age-dating, classification, and spatio-temporal distribution of the SMC clusters
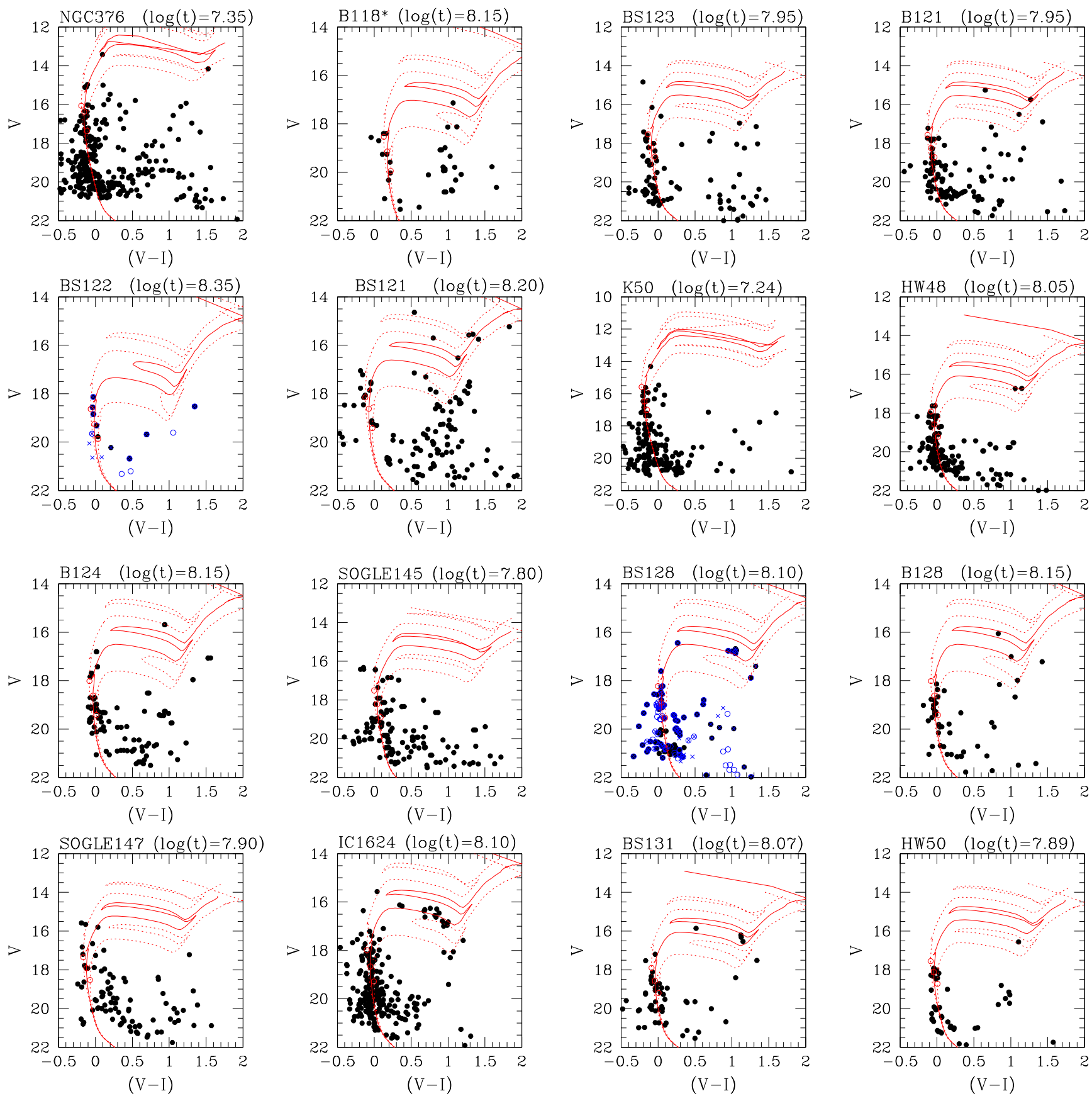

Fig. A.1. continued. 

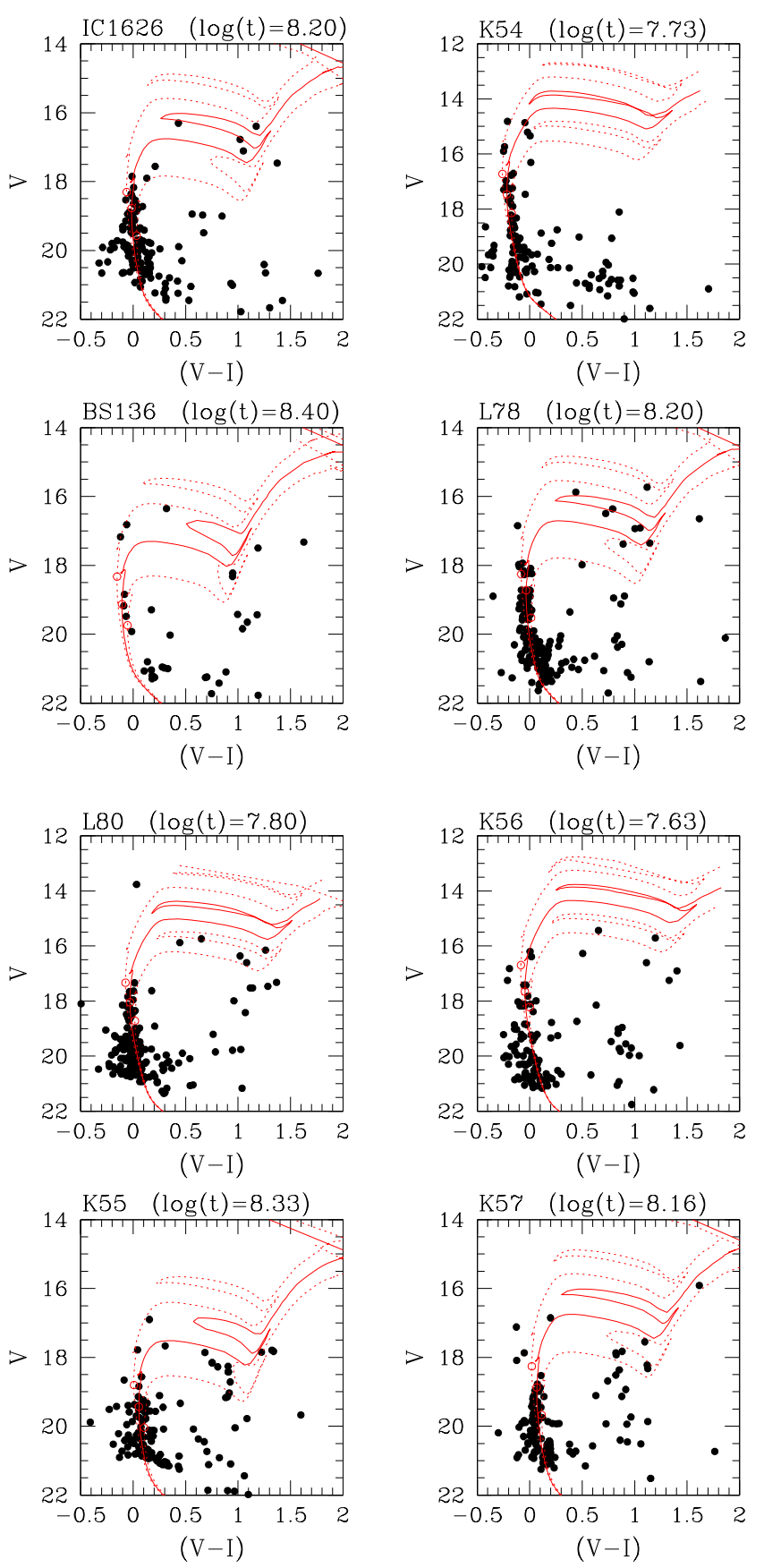

Fig. A.1. continued.

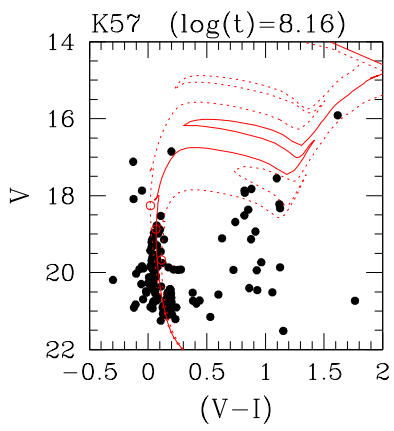

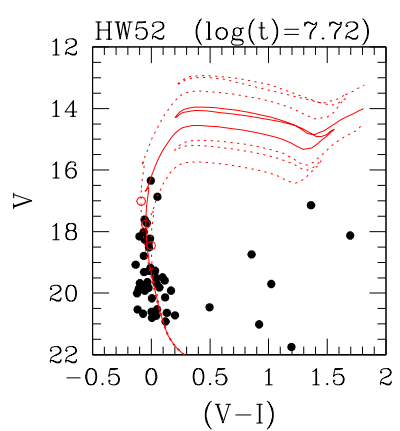
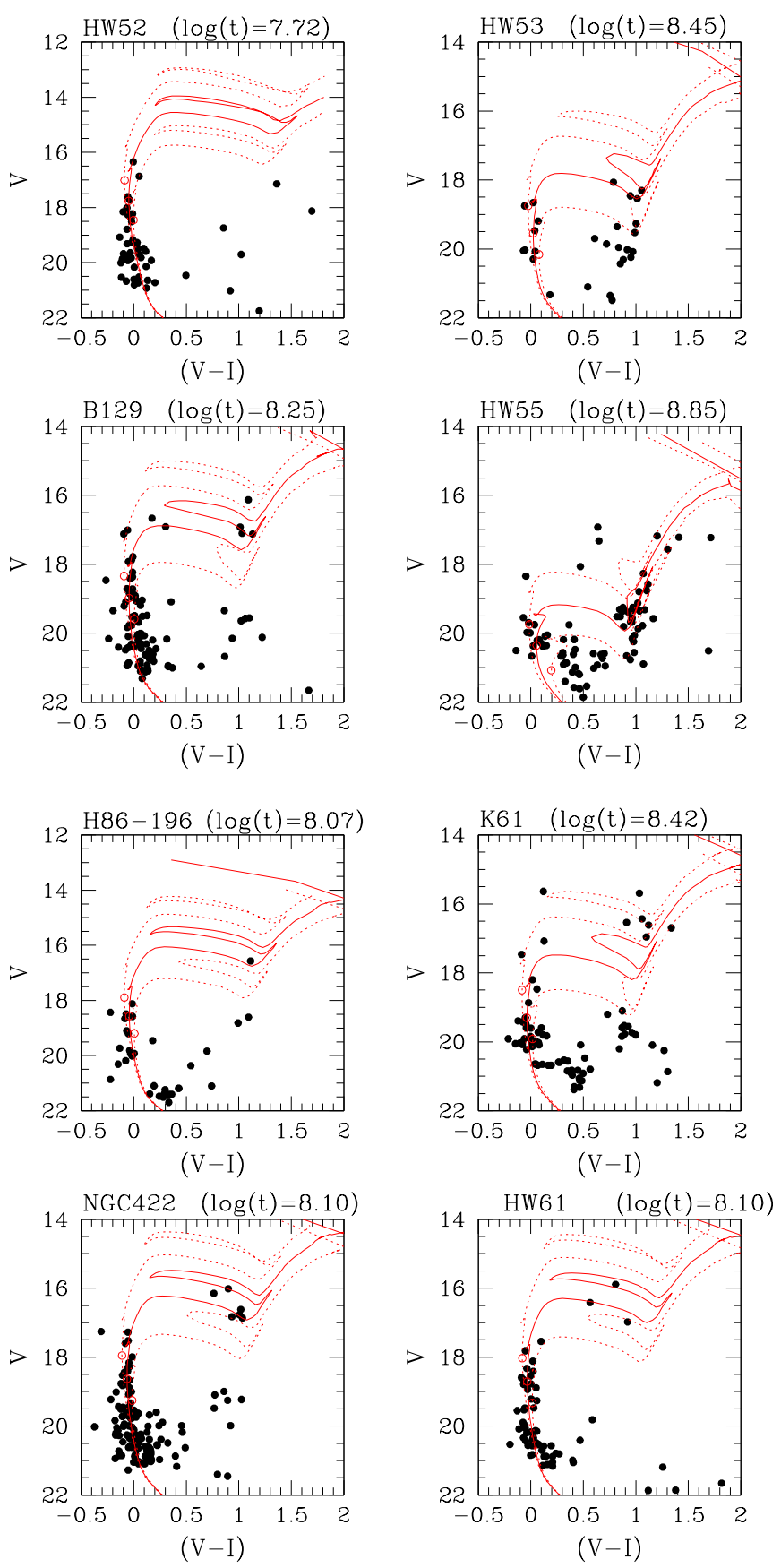
P. K. Nayak et al.: Age-dating, classification, and spatio-temporal distribution of the SMC clusters

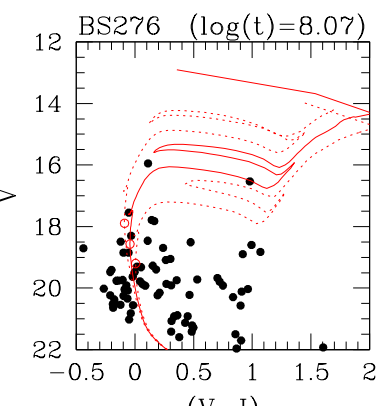

$(\mathrm{V}-\mathrm{I})$
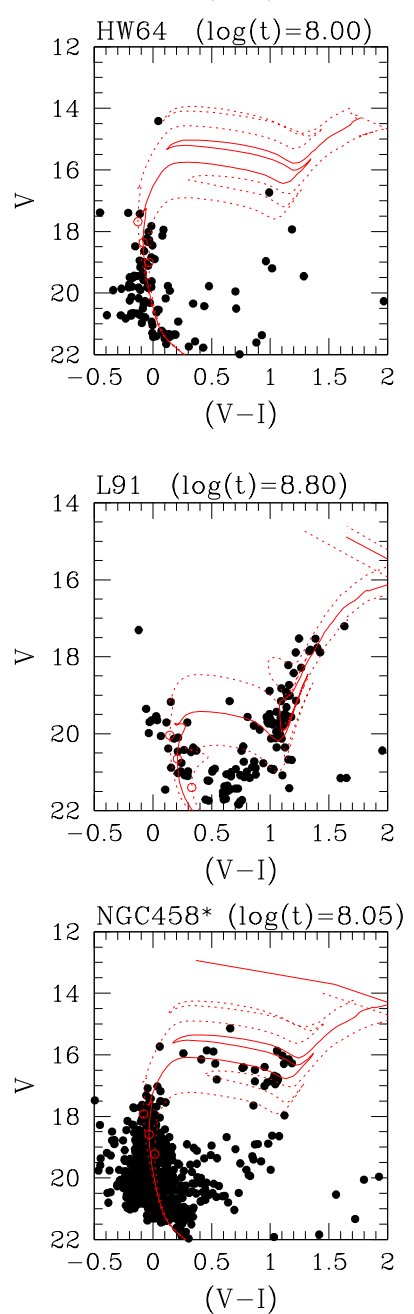
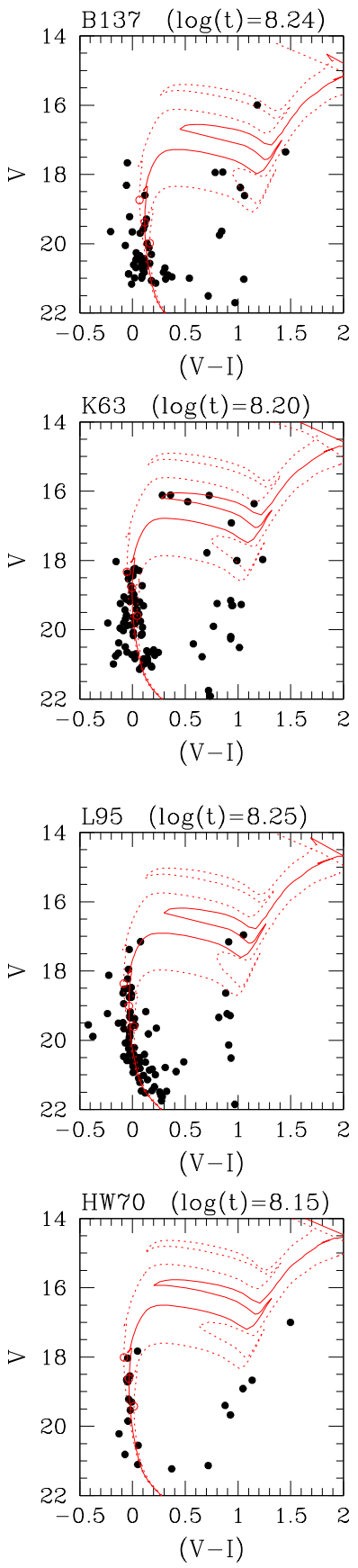

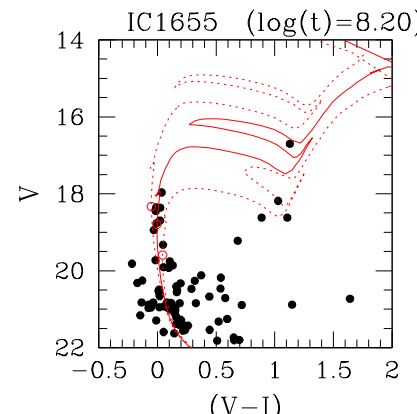

$(\mathrm{V}-\mathrm{I})$
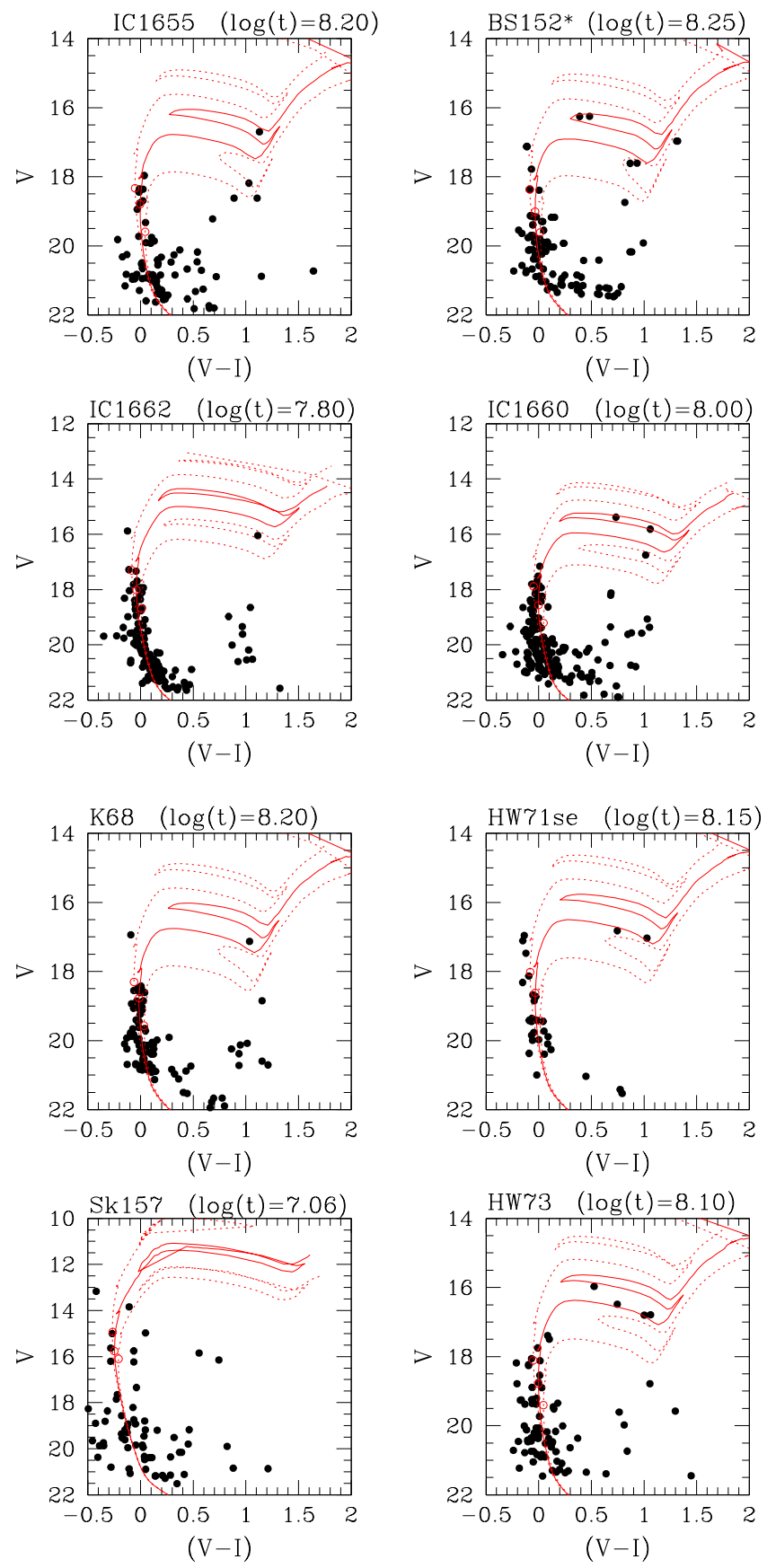

Fig. A.1. continued. 
A\&A 616, A187 (2018)
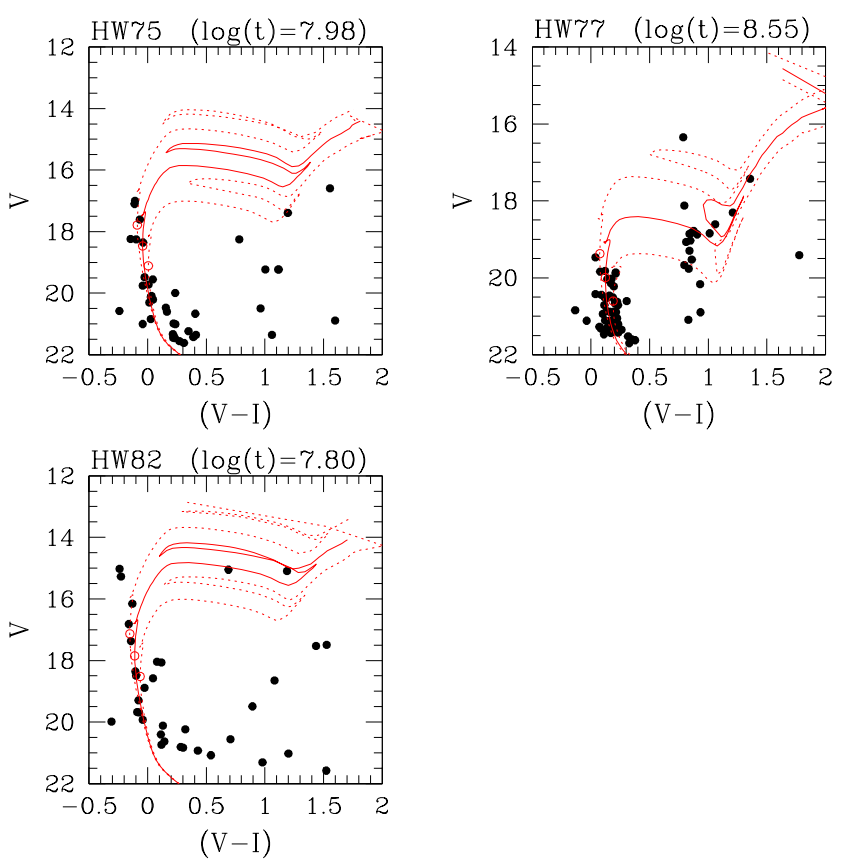

Fig. A.1. continued. 